\title{
Puiseux Series Expansions for the Eigenvalues of Transfer Matrices and Partition Functions from the Newton Polygon Method for Nanotubes and Ribbons
}

\author{
Jeffrey R Schmidt* and Dileep Karanth
}

Department of Physics, University of Wisconsin-Parkside, USA

\begin{abstract}
For certain classes of lattice models of nanosystems the eigenvalues of the row-to-row transfer matrix and the components of the corner transfer matrix truncations are algebraic functions of the fugacity and of Boltzmann weights. Such functions can be expanded in Puiseux series using techniques from algebraic geometry. Each successive term in the expansions in powers a Boltzmann weight is obtained exactly without modifying previous terms. We are able to obtain useful analytical expressions for any thermodynamic function for these systems from the series in circumstances in which no exact solutions can be found.
\end{abstract}

\section{Introduction}

\section{Transfer matrices and partition functions}

The partition function $Z$ for vertex and face models of lattice systems in statistical mechanics (such as those with nearest neighbor interactions) can be computed by application of matrix mathematics to the problem in at least two ways. For models with nearest neighbor interactions only, a row-to-row transfer matrix [1] can be constructed whose eigenvalues determine $Z$. For example let the states of a lattice site $i$ in a row of length $m$ of the lattice be enumerated by a state-variable $t_{i}, 1 \leq i \leq m$. The state-variables of a row in the lattice form a vector $\mathbf{t}=\left(t_{1}, t_{2}, \cdots, t_{m}\right)$, and the Hamiltonian for the system decomposes as

$$
H=\sum_{(\mathbf{t}, \mathbf{t})} h\left(\mathbf{t}, \mathbf{t}^{\prime}\right)+\sum_{\mathbf{t}} k(\mathbf{t})
$$

in which we sum over all pairs $\left(\mathbf{t}, \mathbf{t}^{\prime}\right)$ of adjacent rows. We will use $t$ and $s$ for state-variables appearing in row-to-row transfer matrices, and $a, b$ and $c$ for state-variables appearing in corner transfer matrices.

If the lattice has a periodic boundary condition on its $N$ rows, the partition function is writable in terms of eigenvalues $x_{i}$ of the row-torow transfer matrix $T$,

$$
Z=\sum_{\{\mathbf{t}\}} \sum_{\left\{\mathbf{t}^{\prime}\right\}} \cdots \sum_{\left\{\mathbf{t}^{\prime \prime}\right\}} T_{\mathrm{t}, \mathrm{t}^{\prime}} \cdots T_{\mathbf{t}^{\prime}, \mathrm{t}^{\prime \prime}}=\operatorname{tr} T^{N}=\sum_{i} x_{i}^{N}, \quad T_{\mathbf{t}, \mathbf{t}^{\prime}}=e^{-\beta(h(\mathbf{t}, \mathbf{t})+k(\mathbf{t})+k(\mathbf{t}) 2)}
$$

in which the sums are over all of the possible states of each row. If $N$ is very large, the free energy per site will be determined by a single eigenvalue, the largest, which we will label as $x_{1}, x_{1}>x_{i}$, for $i>1$

$$
f=-\lim _{N \rightarrow \infty} k T \ln Z N=-k T \ln x_{1}-\lim _{N \rightarrow \infty} k T N \ln \left(1+\left(\frac{x_{2}}{x_{1}}\right)^{N}+\cdots\right)=-k T \ln x_{1}
$$

Computation of $N$-point functions $(n \geq 2)$ require additional eigenvalues [1]. The characteristic equation $0=\operatorname{det}(T-X \cdot 1)$ for the row-to-row transfer matrix $T=T(z)$ will be a multi-variable polynomial in $X$ and Boltzmann weights $z$ (or fugacity $z$ if $Z$ is the grand canonical partition function), so the solutions $X=x_{i}$ will be algebraic functions of these weights. It is quite a challenge to compute these eigenvalues exactly in dimensions higher than one.

The most extensively studied two-dimensional lattice models are the $m \times N$ lattice vertex and face models in the thermodynamic limit of both $m, N \rightarrow \infty$, the row-to-row transfer matrix eigenvalues are obtained by the Bethe Ansatz or methods descended from it. A number of interesting cases have not yet been solved exactly (hard squares, the Ising model with external fields, to name two). In such cases series expansions can provide some answers to questions about th physical properties. There is a vast body of literature on series expansions for the row-to row $Z$ and the free energy per particle for interacting lattice models [2], and the subject continues to receive attention [3].

Corner transfer matrices can be used to define a variety of partition functions, on a triangular lattice three partition functions $Z_{A}, Z_{2}$, (Figure 2) and $Z_{w}$ (Supplementary Figure 1 in the appendix), can be constructed $[1,4,5]$ from matrices $A(a), F(a, b)$ which are functions of the state-variables $a, b$ of central sites (we discuss a few details in the appendix);

$$
\begin{aligned}
& Z_{A}=\sum_{a} \operatorname{Tr} A^{6}(a) \\
& Z_{2}=\sum_{a, b} \operatorname{Tr} A^{2}(a) F(a, b) A^{2}(b) F(b, a)
\end{aligned}
$$

(thethird partitionfunction $Z_{w}=\sum_{a, b, c} w(a, b, c) F(a, b) A(b) F(b, c) A(c) F(c, a) A(a)$ is given in the appendix, $w(a, b, c)$ is the weight of the face of the lattice whose vertices are $a, b, c)$ and from these one can express the partition function per lattice site as $\kappa=\frac{Z_{A} Z_{w}^{2}}{Z_{2}^{3}}$ as the lattice size becomes infinite. By taking variations of Boltzmann parameters that leave the partition function per site $\kappa$ unchanged, two corner transfer matrix equations (on the triangular lattice) can be obtained $[4,5]$.

$$
\begin{aligned}
& \sum_{c} w(a, b, c) F(b, c) A(c) F(c, a)=\left(\frac{Z_{w}}{Z_{2}}\right) A(b) F(b, a) A(a) \equiv \eta A(b) F(b, a) A(a) \\
& \sum_{b} F(a, b) A^{2}(b) F(b, a)=\left(\frac{Z_{2}}{Z_{A}}\right) A^{4}(a) \equiv \xi A^{4}(a), \quad \kappa=\frac{\eta^{2}}{\xi}
\end{aligned}
$$

*Corresponding author: Dr. Jeffrey R Schmidt, Associate Professor-Physics, Mathematics and Physics, University of Wisconsin-Parkside, 900 Wood Road, Kenosha, Wisconsin 53141, USA, Tel: 202-537-3645; E-mail: schmidt@uwp.edu

Received July 27, 2013; Accepted February 28, 2014; Published March 10, 2014

Citation: Schmidt JR, Karanth D (2014) Puiseux Series Expansions for the Eigenvalues of Transfer Matrices and Partition Functions from the Newton Polygon Method for Nanotubes and Ribbons. J Phys Math 5: 125 doi: $10.41722090-$ 0902.1000125

Copyright: @ 2014 Schmidt JR, et al. This is an open-access article distributed under the terms of the Creative Commons Attribution License, which permits unrestricted use, distribution, and reproduction in any medium, provided the original author and source are credited. 


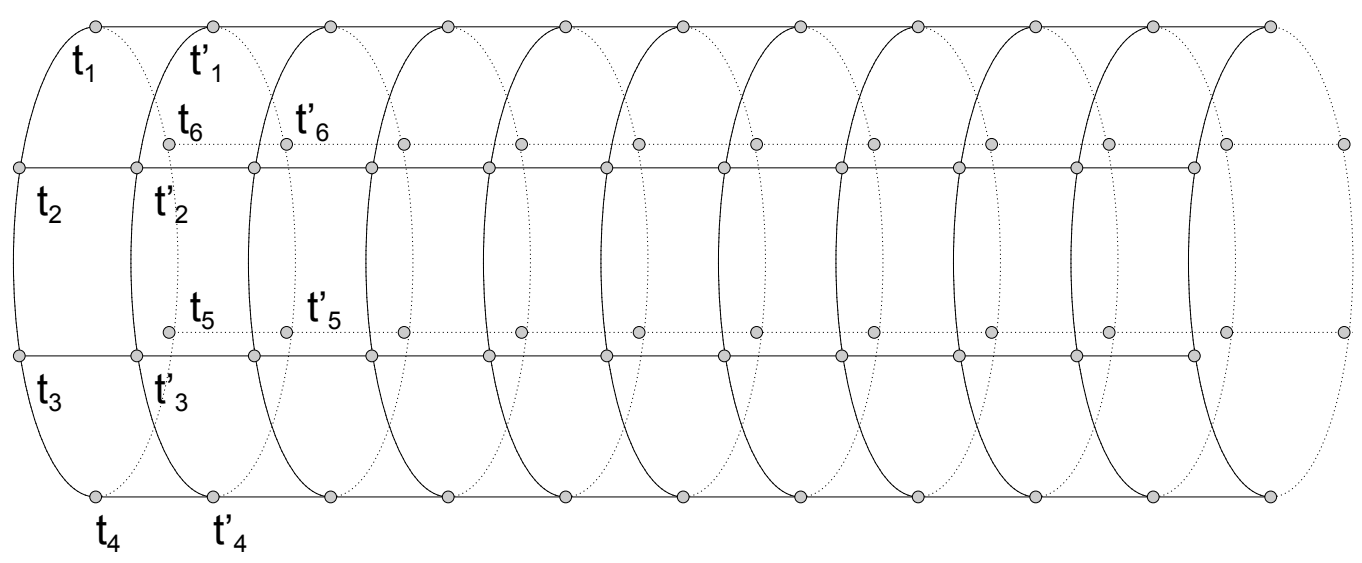

Figure 1: Adjacent rows of sites in a $6 \times N$ Ising model tube.
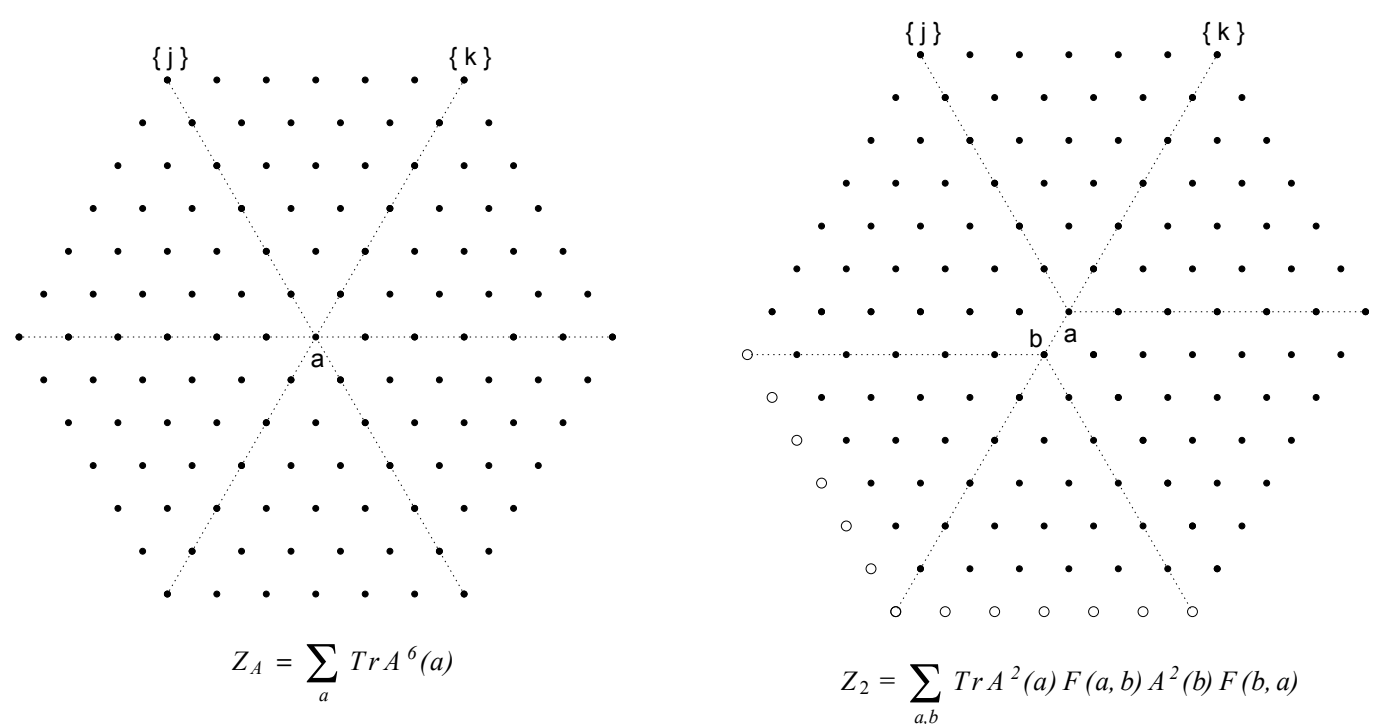

Figure 2: Two partition functions using corner transfer matrices for a triangular lattice.

The square lattice case is a bit more complex, with eight partition functions. Computation of the partition function per site $\kappa$ from the corner transfer matrix equations is even more difficult than the row-to-row matrices because of their nonlinearity. Approximation methods truncate the infinite-dimensional matrices $A(a)$ and $F(a, b)$ to some finite size in representations with $A(a)$ diagonal, and even the most extreme approximation (truncation to one by one matrices, the Kramers-Wannier approximation) gives surprisingly good results for $\kappa$, for a non-interacting lattice gas this approximation is in fact exact.

We have found that for a number of important models on $m \times N$ lattices with $m$ finite and $N \rightarrow \infty$, the eigenvalues of the row-to-row transfer matrix $T$, and the corner transfer-matrix "eigenvalues" $\eta$ and $\xi$ can be expanded in Puiseux series in the Boltzmann weights, by a powerful method from the theory of algebraic geometry, the Newton polygon. This is a type of series expansion that does not appear to have been considered before in statistical physics (but has been used recently in cosmological calculations [6]). These ideas allow us to obtain analytical formulas for thermodynamical quantities for nanosystems such as whiskers, filaments, and tubes, in which one dimension is small and one is large.

Many symbolic-numerical methods for finding eigenvalues of matrices or roots of polynomials can fail on degenerate roots $[7,8]$ but this does not appear to happen for the problems that we investigated by the techniques described in this article. We found the largest root is always non-degenerate (a consequence of the Frobenius-Perron theorem), and we are able to find expansions of degenerate "less significant" roots without any difficulties, in fact there were tell-tale signs at stages in the calculations that revealed what the degeneracies were. The way in which these series expansions are constructed is fundamentally different from traditional developments of power series expansions of functions such as $Z$ and $f$ in that it is not a perturbation or cluster expansion or a counting of graphs. The expansions gotten by successive iterations of the Newton polygon method are not progressive refinements; rather they are extensions, each of which gives one new term in the series exactly, and does not alter preceding terms. 


\section{The Geometry of Algebraic Functions}

We will first introduce the Puiseux series of an algebraic function, and the procedure for obtaining them using the method of the Newton polygon. Next we explain what the series represent, and why the method works the way it does.

\section{Puiseux series}

If $K$ is a field then we can define the closed non-Archimedean field $K[[z]]$ of Puiseux series over $K$ (for our purposes $K=C$ or $R$ ) with coefficients $f_{k} \in K$ as the set of formal expressions of the form [9-11].

$$
\bar{f}(z)=\sum_{k=k_{0}}^{\infty} f_{k} z^{\frac{k}{n}}
$$

where $n$ and $k_{0}$ are a nonzero natural number and an integer respectively (which are part of the datum of $f$ ): in other words, Puiseux series differ from formal Laurent series in that they allow for fractional exponents of the indeterminate as long as these fractional exponents have bounded denominator (here $n$ ), and like Laurent series, Puiseux series allow for negative exponents of the indeterminate as long as these negative exponents are bounded (here by $k_{0}$ ).

This type of series will arise in statistical physics when we seek expansions of the roots (eigenvalues) $X=\bar{x}_{i}, i=1,2, \cdots, p$ of characteristic polynomials of the row-to-row or corner transfer matrix

$$
\operatorname{det}(T(z)-X \cdot 1)=P(X, z)=\bar{a}_{0}(z)+\bar{a}_{1}(z) X+\bar{a}_{2}(z) X^{2}+\cdots \bar{a}_{p}(z) X^{p}=0
$$

which defines $X=\bar{x}_{i}$ as an algebraic function of $Z$ (the fugacity or a Boltzmann weight)

$$
\bar{x}_{i}=x_{i, 1} z^{\xi_{i, 1}}+x_{i, 2} z^{\xi_{i, 1}+\xi_{i, 2}}+x_{i, 3} z^{\xi_{i, 1}+\xi_{i, 2}+\xi_{i, 3}}+\cdots
$$

We introduce some terminology used in algebraic geometry: if $\bar{x}_{i} \in K[[z]]$ with $x_{i, 1} \neq 0$ and with the exponents $\xi_{i}=\sum_{j} \xi_{i, j}$ ordered $\xi_{1}<\xi_{2}<\cdots$, we call the order of $\bar{x}_{i}$ the function $O\left(\bar{x}_{i}\right)=\xi_{i, 1}$ (exponent of the lowest power of the indeterminate), and the initial coefficient of $\bar{x}_{i}$ is $\operatorname{In}\left(\bar{x}_{i}\right)=x_{i, 1}$. These would correspond to the exponents and coefficient of the leading term with respect to a local ordering in the case of polynomials or power series.

The polynomial (in $X)$ Eq. 6 defines an algebraic curve in the $(X, z)$ plane. The problem of parameterizing such a curve can be summarized in a few steps [10]. First a theorem; in some suitable coordinate system any parameterization of the $i^{\text {th }}$ branch is equivalent to one of the form

$$
z=z(t)=t^{n}, \quad X=\bar{x}_{i}=x_{i, 1} t^{n_{1}}+x_{i, 2} t^{n_{2}}+x_{i, 3} t^{n_{3}}+\cdots, \quad 0<n, \quad 0<n_{1}<n_{2}<\cdots
$$

(introducing parameter $t$ ). Fractional power series occur when we eliminate $t$ and put $X \in K\left[z^{1 n}\right] \subset K[[z]]$. The second step is the development of the expansion with respect to some monomial ordering (which is a local ordering in $K[z]$ ). Let $P$ be a polynomial in $X$ (integer powers of $X$ ) with coefficients in $K[[z]]^{*}=\bigcup_{n=1}^{\infty} K\left[z^{\frac{1}{n}}\right]$. Write it as

$$
P(X, z)=\sum_{j=0}^{p} \bar{a}_{j}(z) X^{j}=\bar{a}_{p}(z) \prod_{j=1}^{p}\left(X-\bar{x}_{j}(z)\right), \quad P\left(\bar{x}_{i}, z\right)=0, \quad i=1,2, \cdots, p
$$

Then solutions are of the form Eq. 7 (suppressing the subscript $i$ labeling the branches)

$$
X=c z^{\gamma}+c^{\prime} z^{\gamma+\gamma_{1}}+c^{\prime \prime} z^{\gamma+\gamma_{1}+\gamma_{2}}+\cdots, \quad \gamma=O(X), \quad \gamma_{i}>0
$$

and the series is built term by term. The idea of the proof of this statement is as follows; write $X=z^{\gamma}\left(c+X_{1}(z)\right)$ with $O\left(X_{1}\right)=\gamma_{1}>0$ substitute and expand Eq. 9

$$
P=\left(\bar{a}_{0}(z)+\bar{a}_{1}(z) c z^{\gamma}+\bar{a}_{2}(z) c^{2} z^{2 \gamma}+\cdots+\bar{a}_{p}(z) c^{p} z^{p \gamma}\right)+g\left(X_{1}(z)\right)
$$

This can be zero if the terms with like powers of $z$ cancel; begin with the leading term according to our monomial ordering and let

$$
f=\min \left(O\left(\bar{a}_{j}(z) c^{j} z^{j \gamma}\right)\right)=\min \left(O\left(\bar{a}_{j}\right)+j \gamma\right)
$$

be the lowest power of $z$ appearing on the right side, then if $\operatorname{In}\left(\bar{a}_{\ell}\right)$ is the coefficient of the leading term of $\bar{a}_{\ell}(x)$

$$
\sum_{\ell}^{O\left(\bar{a}_{\ell}(z)\right)+\ell=f} \operatorname{In}\left(\bar{a}_{\ell}\right) c^{\ell}=0
$$

which we solve for $c$. We now iterate the process on what is left over, using the next term in the series solution to kill the next lowest powers of $z$. The lowest powers of $z$ will come from those terms of $P(X, z)$ corresponding to the very lowest points of the Newton diagram of $P(X, z)$.

\section{The Newton diagram of $P(X, z)$}

The algorithm for carrying out this construction of the series is called Newton's method. The first term $c z^{\gamma}=x_{i, 1} z^{\xi_{i, 1}}$ of the series can be found by purely geometric considerations, the next term will be obtained by applying Newton's method to the remainder after the leading term elimination; $P\left(X+x_{i, 1} z^{\xi_{i, 1}}, z\right)=0$, and higher terms computed by further iterations $[11,12]$.

For small $z$, candidates for the dominant term are found geometrically. For every term $c X^{i} z^{r}$ in $P(X, z)$ draw a dot $(i, r)$ in the $i \in \mathbb{Z}, r \in \mathbb{Q}$ plane. These constitute the Newton diagram of $P(X, z)$, which will be columns of dots at integer intervals horizontally. The Newton polygon is the convex hull of the dots at the bottom of the columns. The Newton polygon will give us the numbers $x_{1}$ and $\xi_{1}$ in the Puiseux expansion of the roots above. For example [11] the polynomial

$$
P(X, z)=z-2 z^{2} X^{2}-X^{3}+z X^{4}+z X^{5}
$$

has Newton diagram consisting of the points $\{(0,1),(2,2),(3,0),(4,1),(5,1)\}$ (Figure 3). To create the convex lower hull, find the set of points at the bottom of each column, which in this case is all of the points. Order them by increasing first component to get points $\left\{M_{0}, M_{1}, \cdots\right\}$, and draw a vertical line downwards from $M_{0}$ (in this case $\left.(0,1)\right)$ to serve as an

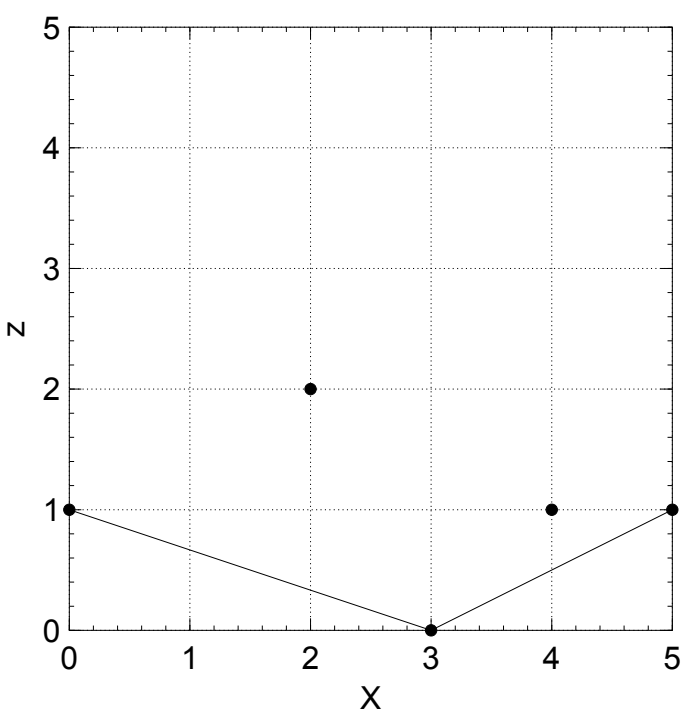

Figure 3: Newton diagram of Eq. 14 
indicator as to how the next line is drawn. You must always turn left. Draw a connecting line from $M_{0}$ to $M_{i}$ if the vertical line from $M_{0}$ downwards hits $M_{i}$ as it is slowly rotated counterclockwise. Then repeat the process but now the indicator line that you rotate lies along $\overline{M_{0} M_{i}}$. There are systematic algorithms, we state the Jarvis Gift Wrap algorithm [13]: create the vectors $(0,0)-M_{0}$, and $M_{i}-M_{0}$, in this case these are $(0,-1)$ and $\{(2,1),(3,-1),(4,0),(5,0)\}$. Now compute the angle between the line connecting the previous two points of the polygon with the last point added to the polygon, and each of the remaining lowest points in each column of points, and select as the next point the one that gives the smallest positive value of this angle. This will ensure that every point of the Newton diagram lies on or above the Newton polygon. The algorithm may not be the most efficient, but it is simple to program.

Once the Newton polygon is found, the algorithm for construction of the roots of Eq. 6 is as follows [11]; each segment $E=\left[M, \cdots, M^{\prime}\right]$ of the Newton polygon of $P$ corresponds to a new homogeneous subpolynomial (a slight departure from the notation of [11], we want to display the $z$-dependence) $Q$ of $P$ of the form

$$
Q(P, E, X, z)=\sum_{h} \operatorname{In}\left(\bar{a}_{h}\right) z^{O\left(\overline{(}_{h}\right)} X^{h}, \quad M_{h} \in E, \quad M_{h}=\left(h, \frac{m_{h}}{m}\right), \quad O\left(\bar{a}_{h}\right)=\frac{m_{h}}{m}
$$

with $h$ running over the exponents of $X$ for points of the Newton diagram on the corresponding segment. The substitution $X=c z^{\gamma}=x_{1} z^{\xi_{1}}$ for a unique $\gamma$ lets one factor out $z$ from $Q$, transforming Eq. 15 into Eq. 13 , to be solved for $C$. [10,11], we have only tried to summarize the basic ideas that distinguish the series from the more familiar series such as Taylor-Maclaurin. The statement in theorem form Basu et al. [11] is; let $\bar{x}$ be a nonzero root of one of these characteristic polynomials $Q$ of a segment $E$ with slope $-\xi_{1}$, of multiplicity $r$, then a Puiseux series for a root of $P$ starting with $x_{1} z^{\xi_{1}}$ is

$$
\bar{x}=x_{1} z^{\xi_{1}}+x_{2} z^{\xi_{1}+\xi_{2}}+\cdots, \quad P(\bar{x}, z)=0
$$

We find that this first term alone is a very good approximation to the roots. The two Newton polynomials for the polynomial $P(X, z)$ of Eq. 14, [11]

$$
Q=z-X^{3}, \quad Q=X^{3}\left(z X^{2}-1\right)
$$

(the two segments in Figure 1) which have roots $X=\bar{x}=1 \cdot z^{\frac{1}{3}}, e^{\frac{2 \pi i}{3}} \cdot z^{\frac{1}{3}}, e^{\frac{4 \pi i}{3}} \cdot z^{\frac{1}{3}}$ and $X= \pm 1 \cdot z^{\frac{1}{2}}$, and slopes $\xi=-1 / 3$ and $1 / 2$, so that the roots of the polynomial have Puiseux series beginning with

$$
\bar{x}=z^{\frac{1}{3}}, \quad e^{\frac{2 \pi i}{3}} z^{\frac{1}{3}}, \quad e^{\frac{4 \pi i}{3}} z^{\frac{1}{3}}, \quad \pm z^{-\frac{1}{2}}
$$

The first term in the Puiseux series expansions for the eigenvalues of the transfer matrix can be computed from a small fraction of the total number of terms in the secular equation, all we need is the lower convex hull. This means that the first terms can be computed by hand even for fairly large transfer matrices. Obtaining the secular (characteristic) equation computationally is more intensive than computing the first terms for the Puiseux series of its roots. A few words are said about this step in the appendix.

\section{Iteration}

Suppose that we start with a transfer matrix secular equation Eq. 9 with $z$ a Boltzmann weight, and obtain the Newton polygon solutions (the first terms of the series expansions) $\bar{x}$ for $X$

$$
\bar{x}=\left\{x_{1,1} z^{\xi_{1,1}}, x_{2,1} z^{\xi_{2,1}}, \cdots, x_{p, 1} z^{\xi_{p, 1}}\right\}
$$

If one performs the substitution

$$
X \rightarrow X+x_{i, 1} z^{\xi_{i, 1}}, \quad P^{\prime}=P\left(X+x_{i, 1} z^{\xi_{i, 1}}, z\right)
$$

and applies the polygon procedure to $P^{\prime}$, one obtains the next term in the Puiseux series for this solution, of the form seen in Eq. 16. This process is now repeated until the series attains the desired precision, in other words for the $i^{\text {th }}$ solution

$$
X \rightarrow X+x_{i, 2} z^{\xi_{i, 1}+\xi_{i, 2}}, \quad P^{\prime \prime}=P^{\prime}\left(X+x_{i, 2} z^{\xi_{i, 1}+\xi_{i, 2}}, z\right)
$$

results in the next correction $x_{i, 3} z^{\xi_{i, 1}+\xi_{i, 2}+\xi_{i, 3}}$.

The calculations are straightforward, but there can be vast numbers of terms in these polynomials. We use the REDUCE computer algebra system [14] to perform the calculations. Singular [15] also has a Puiseux expansion (Hamburger-Noether expansion) library "hnoether.lib", but we prefer our own in REDUCE because we have designed it to make it easier to follow a specific branch. Our REDUCE program NPplus. red returns a list of Newton polygon lines $\{$ slope,intercept, $Q(x, z)\}$. We illustrate its use by expanding the roots of the multivariate polynomial

$$
P=X^{3}-4 X z+z^{2}
$$

(in each iteration we follow the top of first branch $=Q(X, z)$ selection)

$$
\begin{aligned}
& \text { First iteration } \quad P(X, z)=X^{3}-4 X z+z^{2} \rightarrow\left\{\begin{array}{l}
Q(X, z)=X^{3}-4 X z \\
Q(X, z)=z^{2}-4 X z
\end{array}\right. \\
& x_{1,1} z^{\xi_{1,1}}=2 z^{\frac{1}{2}} \\
& \text { Second iteration } \quad P(X, z)=P(X+2 \sqrt{z}, z) \rightarrow\left\{\begin{array}{l}
Q(X, z)=z^{2}+8 X z \\
Q(X, z)=X^{3}+8 X z+6 X^{2} \sqrt{z}
\end{array}\right. \\
& x_{1,2} z^{\xi_{1,1}+\xi_{1,2}}=-\frac{1}{8} z \\
& \text { Third iteration } P(X, z)=P(X-z / 8, z) \rightarrow\left\{\begin{array}{l}
Q(X, z)=\frac{3}{32} z^{\frac{5}{2}}+8 X z \\
Q(X, z)=X^{3}+8 X z+6 X^{2} \sqrt{z}
\end{array}\right. \\
& x_{1,3} z^{\xi_{1,1}+\xi_{1,2}+\xi_{1,3}}=-\frac{3}{256} z^{\frac{3}{2}} \\
& \text { Fourth iteration } \quad P(X, z)=P\left(X-\frac{3}{256} z^{\frac{3}{2}}, z\right) \rightarrow\left\{\begin{array}{l}
Q(X, z)=8 X z+\frac{1}{64} z^{3} \\
Q(X, z)=X^{3}+8 X z+6 X^{2} \sqrt{z}
\end{array}\right.
\end{aligned}
$$

from which we deduce that a Puiseux expansion of one of the roots of this polynomial is

$$
\bar{x}_{1}=2 z^{\frac{1}{2}}-\frac{1}{8} z-\frac{3}{256} z^{\frac{3}{2}}-\frac{1}{512} z^{2}+\cdots
$$

and a simple test for numerical values $0 \leq z \leq 0.5$ shows that these few terms give a precision in the value of a root of five decimal places

$$
\bar{x}_{1}^{3}-4 z \bar{x}_{1}+z^{2}=0.00320434570313 z^{\frac{7}{2}}+\mathcal{O}\left(z^{4}\right)
$$

The series that are being obtained are not expansions about a point, like a Taylor-Maclaurin or Laurent series, but are instead series that are the solutions to an algebraic equation correct to a particular order in $z$. These series may actually truncate or terminate, we will see this occur in our study of $m \times N$ Ising models. It should be pointed out that these series solutions are formal, and so are very useful as generating functions for combinatorial data, but convergence under substitution of explicit $z$-values is not guaranteed. As we see here and will see again shortly, convergence for sufficiently large/small $z$-values is actually quite spectacular

\section{Application to $m \times N$ Lattice Gases with Exclusion}

Now we turn our attention to the application of these ideas to statistical physics. The procedure described in the previous section is perfectly suited to computation of high and low temperature expansions for such systems as planar lattice gas ribbons and tubes; the 
former being a lattice gas on an $m \times N$ lattice with periodic boundary conditions in the "long dimension" $N$ only, and the latter case with periodic boundary conditions on both "long" and "short" dimensions.

\section{Hard squares at low fugacity $z<<1$}

Consider the case of hard squares, a square lattice with site occupancy $t_{i}=0,1$ such that no two adjacent sites can be simultaneously occupied. This model has not yet been solved exactly, but in the thermodynamic limit $(N \times N$ lattice, $N \rightarrow \infty)$ corner transfer matrices provide us with some information $[5,16]$. On a square lattice $\kappa=\eta \xi$ is the partition function per particle

$$
\begin{aligned}
& \sum_{b=0,1} F(a, b) A^{2}(b) F(b, a)=\xi A^{2}(a) \\
& \sum_{c, d} w(a, c, d, b) F(a, c) A(c) F(c, d) A(d) F(d, b)=\eta A(a) F(a, b) A(b)
\end{aligned}
$$

For hard squares the face weight function is

$$
w(a, b, c, d)=z^{(a+b+c+d) / 4} \varepsilon(a, b) \varepsilon(b, c) \varepsilon(c, d) \varepsilon(d, a), \quad \varepsilon(a, b)=1-a b
$$

In the Kramers-Wannier approximation one treats the matrices as numbers (one by one matrices), the equations Eq. 25 simplify to $A(0)=1, A(1)=A, \quad F(1,1)=0, \quad F(0,1) \cdot F(1,0)=\xi A^{2}, \quad F^{2}(0,0)=\xi\left(1-A^{4}\right)$, $\eta=\kappa \xi$, elimination of $\eta$ and $\xi$ results in

$$
\begin{aligned}
& \kappa=2 z^{\frac{1}{4}} A^{3}+\left(1-A^{4}\right) \\
& \kappa A=z^{\frac{1}{4}}\left(1-A^{4}\right)+z^{\frac{1}{2}} A^{3}
\end{aligned}
$$

Eliminate $\kappa$ and apply the Newton polygon method to expand $A$ in a Puiseux series in $z$

$A=z^{\frac{1}{4}}\left(1-z+4 z^{2}-21 z^{3}+125 z^{4}-800 z^{5}+5368 z^{6}-37240 z^{7}+264828 z^{8}-1919690 z^{9}+\cdots\right)(2$

and use the first of Eqs. 27 to get the partition function per particle [16]

$\kappa=\lim _{N \rightarrow \infty} Z^{\frac{1}{N^{2}}}=1+z-2 z^{2}+8 z^{3}-40 z^{4}+225 z^{5}-1362 z^{6}+8670 z^{7}-57254 z^{8}+388830 z^{9}+\cdots(2$

where agreement of this result with computer calculations that perform graph counting is discussed. Since the hard squares model has not been solved analytically, series provide the only information available for the thermodynamic limit case.

For a $m \times N$ hard-square ribbon lattice gas with $m$ finite and $N \rightarrow \infty$ we order the (row to row) transfer matrix rows and columns as

$$
T_{1+t_{1}+2 t_{2}+\cdots+2^{m-1} t_{m}, 1+t_{1}^{\prime}+2 t_{2}^{\prime}+\cdots+2^{m-1} t_{m}^{\prime}}=\left(\prod_{j=1}^{m}\left(1-t_{j} t_{j}^{\prime}\right)\left(1-t_{j} t_{j+1}\right)\left(1-t_{j}^{\prime} t_{j+1}^{\prime}\right)\right) z^{\sum_{i=1}^{m}\left(t_{i}+t_{i}^{\prime}\right) / 2}
$$

and solve for the largest eigenvalue $X=x_{\max }$ of polynomial $P(X, z)=\operatorname{det}(T(z)-X \cdot 1)$ using our simple Newton polygon library. Our results for the $N \rightarrow \infty$ partition function per lattice site for the $4 \times N$ ribbon and tube, respectively

$$
\begin{aligned}
& Z_{4 \times N, r i b}^{1 / N}=x_{\max }=1+4 z-z^{2}+8 z^{3}-39 z^{4}+206 z^{5}-1152 z^{6}+6710 z^{7}-40277 z^{8}+\cdots \\
& \kappa=Z^{\frac{1}{4 N}}=1+z-\frac{7}{4} z^{2}+\frac{25}{4} z^{3}-\frac{899}{32} z^{4}+\frac{4557}{32} z^{5}-\frac{99441}{128} z^{6}+\cdots \\
& Z_{4 \times N, \text { tube }}^{1 / N}=x_{\max }=1+4 z-2 z^{2}+12 z^{3}-62 z^{4}+352 z^{5}-2122 z^{6}+13344 z^{7}-86566 z^{8}+\cdots \\
& \kappa=Z^{\frac{1}{4 N}}=1+z-2 z^{2}+8 z^{3}-\frac{159}{4} z^{4}+\frac{885}{4} z^{5}+\frac{2639}{2} z^{6}+\cdots
\end{aligned}
$$

It is reassuring to see that this is a good match for the $N \times N$ case in thermodynamic limit, at least for the lowest order terms. For the $5 \times N$ ribbon and tube respectively we obtain

$$
\begin{aligned}
& Z_{5 \times N, r i b}^{1 / N}=x_{\max }=1+5 z+z^{2}+7 z^{3}-37 z^{4}+208 z^{5}-1225 z^{6}-7485 z^{7} ; \cdots \\
& \kappa=Z^{\frac{1}{5 N}}=1+z-\frac{9}{5} z^{2}+\frac{33}{5} z^{3}-\frac{762}{25} z^{4}+159 z^{5}-\frac{111814}{125} z^{6}+\cdots
\end{aligned}
$$

$$
\begin{aligned}
& Z_{5 \times N, \text { tube }}^{1 / N}=x_{\max }=1+5 z+10 z^{3}-55 z^{4}+325 z^{5}-2025 z^{6}+13120 z^{7}-87545 z^{8}+\cdots \\
& \kappa=Z^{\frac{1}{5 N}}=1+z-2 z^{2}+8 z^{3}-40 z^{4}+\frac{1124}{5} z^{5}-\frac{6791}{5} z^{6}+\cdots
\end{aligned}
$$

For the $6 \times N$ ribbon and tube, respectively

$$
\begin{aligned}
& Z_{6 \times N, r i b}^{1 / N}=x_{\max }=1+6 z+4 z^{2}+6 z^{3}-32 z^{4}+190 z^{5}-1164 z^{6}+7352 z^{7}-47630 z^{8} ; \cdots \\
& \kappa=Z^{\frac{1}{16 N}}=1+z-\frac{11}{6} z^{2}+\frac{41}{6} z^{3}-\frac{2309}{72} z^{4}+\frac{4081}{24} z^{5}-\frac{1260845}{1296} z^{6}+\cdots \\
& Z_{6 \times N, \text { tube }}^{1 / N}=x_{\max }=1+6 z+3 z^{2}+8 z^{3}-45 z^{4}+276 z^{5}-1760 z^{6}+11610 z^{7}-78681 z^{8}+\cdots \\
& \kappa=Z^{\frac{1}{16 N}}=1+z-2 z^{2}+8 z^{3}-40 z^{4}+225 z^{5}-\frac{8171}{6} z^{6}+\frac{51997}{6} z^{7}-\frac{171577}{3} z^{8}+\cdots
\end{aligned}
$$

(note that six must be a good approximation to infinity for eighth order; the $z^{8}$ term agrees with the corner transfer matrix result for $N \times N, N \rightarrow \infty$ to within 11 parts in 10,000). We can go on

$$
\begin{aligned}
& Z_{7 \times N, \text { the }}^{1 / N}=1+7 z+7 z^{2}+7 z^{3}-35 z^{4}+224 z^{5}-1463 z^{6}+9807 z^{7}-67270 z^{8}+470442 z^{9}+\cdots \\
& \kappa=Z^{\frac{1}{17 N}}=1+z-2 z^{2}+8 z^{3}-40 z^{4}+225 z^{5}-1362 z^{6}+\frac{60689}{7} z^{7}-\frac{400744}{7} 7 z^{8}+\cdots \\
& Z_{8 \times N, \text { the }}^{1 / N}=1+8 z+12 z^{2}+8 z^{3}-26 z^{4}+176 z^{5}-1180 z^{6}+8048 z^{7}-55886 z^{8}+394488 z^{9}+\cdots \\
& \kappa=Z^{\frac{1}{18 N}}=1+z-2 z^{2}+8 z^{3}-40 z^{4}+225 z^{5}-1362 z^{6}+8670 z^{7}-\frac{458023}{8} z^{8}+\cdots
\end{aligned}
$$

REDUCE is built for comfort, not for speed, nevertheless each iteration takes only a second or two even for the $64 \times 64$ transfer matrix of the $6 \times N$ case.

\section{Hard squares at high fugacity $z>>1$}

The high fugacity expansions for the lattice gases do in general result in true Puiseux series, with both positive and negative fractional exponents. For the simplest case, which can be worked out by hand, the characteristic polynomial for the $1 \times N$ hard-squares tube is

$$
T_{1+t_{1}, 1+t_{1}^{\prime}}=\left(1-t_{1} t_{1}^{\prime}\right) z^{\left(t_{1}+t_{1}^{\prime}\right) / 2}, \quad P(X, z)=\operatorname{det}(T-X \cdot 1)=X^{2}-X-z
$$

To obtain the high-fugacity expansion we transform $z \rightarrow 1 u$, and multiply the entire polynomial by a sufficient number $q$ of $u$ factors (the order of $P(x, z)$ as a function of $z$ ) in order to make all $u$-exponents positive;

$$
P(x, z) \rightarrow u^{q} P\left(x, u^{-1}\right)=u P\left(X, u^{-1}\right)=u X^{2}-u X-1
$$

and apply the Newton construction, the largest eigenvalue will be seeded by the lowest power of $u$, and finally perform $u \rightarrow z^{-1}$ to obtain

$$
\begin{aligned}
& Z_{1 \times N}^{1 / N}=1+z-z^{2}+2 z^{3}-5 z^{4}+14 z^{5}-42 z^{6}+132 z^{7}-429 z^{8}+\cdots \quad z<<1 \\
& Z_{1 \times N}^{1 / N}=z+\frac{1}{2}+\frac{1}{8} z^{-\frac{1}{2}}-\frac{1}{128} z^{-\frac{3}{2}}+\frac{1}{1024} z^{-\frac{5}{2}}+\cdots \quad z>>1
\end{aligned}
$$

We get a better picture of what transformation Eq. 36 does for the $3 \times N$ hard squares tube, compare the Newton diagrams (Figure 4) in each regime

For $z<<1$ the characteristic polynomial is

$$
\begin{aligned}
& P(X, z)=X^{4}\left(X^{4}-X^{3}-3 X^{2}\left(z^{2}+z\right)\right. \\
& \left.-X\left(2 z^{3}+3 z^{2}\right)-z^{3}\right)
\end{aligned}
$$

and for $u=1 z<<1$

$$
\begin{aligned}
& u^{3} P(X, u)=X^{4}\left(X^{4} u^{3}-X^{3} u^{3}-3 X\left(u^{2}+u\right)\right. \\
& -X(3 u+2)-1)
\end{aligned}
$$

The lowest level construction results in $\bar{x}=1$ and a doublydegenerate $-z$ for the former case and $\bar{x}=-12,-u^{-1},-u^{-1}, 2 u^{-1}$ for the latter. Since the Puiseux series solutions Eq. 7 have ascending positive 

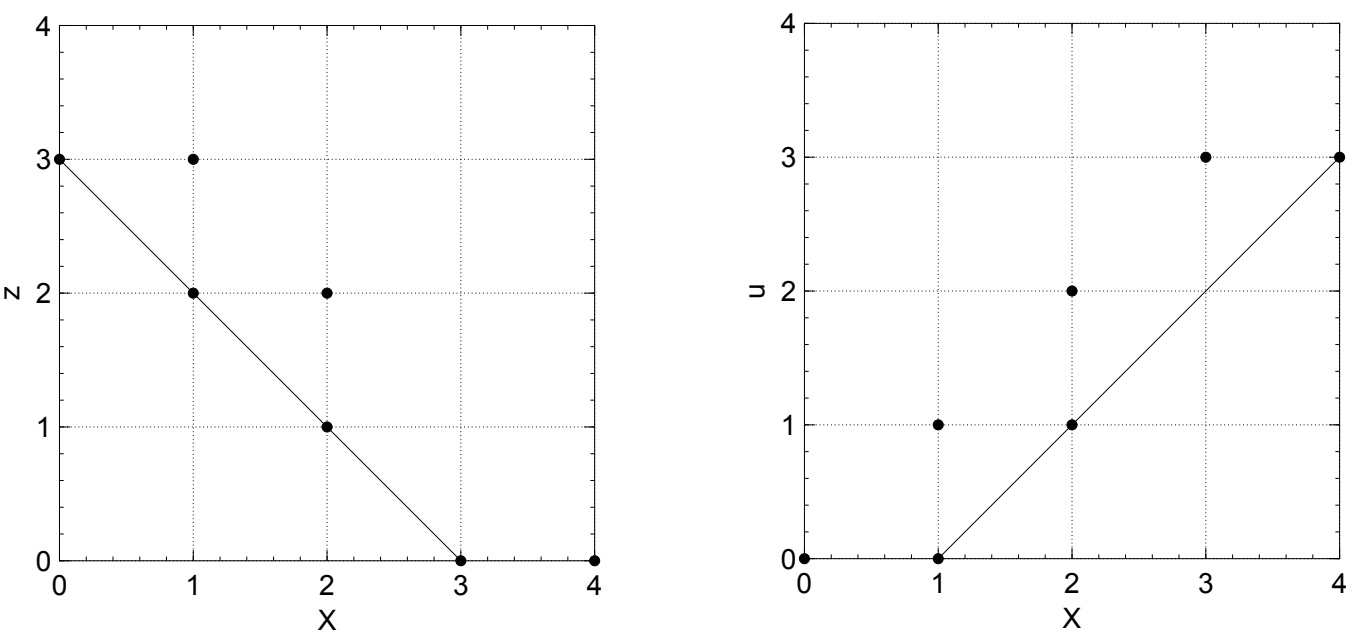

Figure 4: $P(x, z) \rightarrow u^{q} P\left(x, u^{-1}\right)$ is a symmetry transforming upper convex hull of one diagram into lower convex hull.

exponents, when $z<<1$ the solution starting with $\bar{x}=1$ will be largest, and when $u^{-1}=z>>1$ or $u<<1$ the series starting with $2 u^{-1}$ will be largest;

$$
\begin{aligned}
& Z_{3 \times N, \text { tube }}^{1 / N}=\frac{2}{u}+\frac{3}{2}-\frac{3}{8} u+\frac{3}{8} u^{2}-\frac{57}{128} u^{3}+\frac{75}{128} u^{4}-\frac{843}{1024} u^{5}+\cdots \\
& =2 z+\frac{3}{2}-\frac{3}{8} z^{-1}+\frac{3}{8} z^{-2}-\frac{57}{128} z^{-3}+\frac{75}{128} z^{-4}-\frac{843}{1024} z^{-5}+\cdots \quad z>>1 \\
& Z_{3 \times N \text {,tube }}^{1 / N}=1+3 z-3 z^{2}+12 z^{3}-57 z^{4}+300 z^{5}+1686 z^{6}+\cdots \quad z<<1 \\
& Z_{4 \times N, \text { thbe }}^{11 N}=z^{2}+2 z+4-2 z^{-1}-8 z^{-2}+22 z^{-3}-190 z^{-5}+120 z^{-6}+1442 z^{-7}-3268 z^{-8}, \quad z>>1 \\
& Z_{4 \times N, \text {,ube }}^{1 / N}=1+4 z-2 z^{2}+12 z^{3}-62 z^{4}+352 z^{5}-2122 z^{6}+13344 z^{7}-86566 z^{8}+\cdots \quad z<<1 \\
& Z_{5 \times N, \text {,ube }}^{1 / N}=1+5 z+10 z^{3}-55 z^{4}+325 z^{5}-20225 z^{6}+13120 z^{7}-87545 z^{8}+\cdots, z<<1 \\
& Z_{\text {SאN, tube }}^{1 / N}=2 z^{2}+\frac{9}{2} z+\frac{11}{8}-\frac{15}{16} z^{-1}+\frac{339}{128} z^{-2}-\frac{1765}{256} z^{-3}+\frac{16831}{1024} z^{-4}-\frac{72975}{2048} z^{-5}+\cdots z>>1 \\
& Z_{6 \times N, \text {,ube }}^{1 / N}=1+6 z+3 z^{2}+8 z^{3}-45 z^{4}+276 z^{5}-1760 z^{6}+11610 z^{7}-78681 z^{8}+\cdots, \quad z<<1 \\
& Z_{6 \times N, \text { thbe }}^{1 / N}=z^{3}+3 z^{2}+3 z+14-9 z^{-1}+45 z^{-2}-371 z^{-3}+1134 z^{-4}-4959 z^{-5}+27798 z^{-6} \\
& -121002 z^{-7}+570303 z^{-8}+\cdots \quad z>>1 \\
& Z_{8 \times N, \text {,ube }}^{1 / N}=1+8 z+12 z^{2}+8 z^{3}-26 z^{4}+176 z^{5}-1180 z^{6}+8048 z^{7}-55886 z^{8}+39448 z^{9}+\cdots \quad z<<1 \\
& Z_{8 \times N, \text { tube }}^{1 / N}=z^{4}+4 z^{3}+6 z^{2}+8 z+44-12 z^{-1}+166 z^{-2}-612 z^{-3}-1444 z^{-4}+\cdots \quad z>>1
\end{aligned}
$$

We take a moment to address convergence. These formulas are in excellent agreement with high-precision numerical computation of the eigenvalues (obtained using Gnu octave).

\begin{tabular}{|l|c|c|c|c|}
\hline & \multicolumn{2}{|c|}{ HS $5 \times N$ tube } & \multicolumn{2}{c|}{ HS $6 \times N$ tube } \\
\hline$z$ & $\bar{x}_{\max }$ (octave) & $\bar{x}_{\max }$ (Eq. 40) & $\bar{x}_{\max }$ (octave) & $\bar{x}_{\max }$ (Eq. 40) \\
\hline 0.01 & 1.05000948 & 1.05000946 & 1.06030757595 & 1.06030757595 \\
\hline 0.025 & 1.12513751 & 1.12513307 & 1.15198475 & 1.15198475 \\
\hline 0.05 & 1.25098387 & 1.25069862 & 1.30828428 & 1.30828350 \\
\hline 5 & $7.37564404 \mathrm{e}+01$ & $7.37575383 \mathrm{e}+01$ & $2.27193463 \mathrm{e}+02$ & $2.27949742 \mathrm{e}+02$ \\
\hline 10 & $2.46302186 \mathrm{e}+02$ & $2.46302195 \mathrm{e}+02$ & $1.34326223 \mathrm{e}+03$ & $1.34326421 \mathrm{e}+03$ \\
\hline 20 & $8.91333977 \mathrm{e}+02$ & $8.91333977+02$ & $9.27362202 \mathrm{e}+03$ & $9.27362202 \mathrm{e}+03$ \\
\hline 40 & $3.38135312 \mathrm{e}+03$ & $3.38135312 \mathrm{e}+03$ & $6.89337977288 \mathrm{e}+04$ & $6.89337977288 \mathrm{e}+04$ \\
\hline
\end{tabular}

The last of Eqs. 40 are approaching the infinite lattice results for $z \gg>1$ reported in Appendix B of [4] computed by numerical computations of corner transfer matrix truncations, since for the $m \times n$ lattice $Z_{m \times N}^{1 / N}=\kappa^{m}$

$$
\begin{aligned}
& z^{-1} \kappa^{2}\left(z^{-1}\right)=1+z^{-1}+z^{-3}-z^{-4}+5 z^{-5}-10 z^{-6}+39 z^{-7}-95 z^{-8} \\
& \kappa^{6}=z^{3}+3 z^{2}+3 z+4+3 z^{-1}+12 z^{-2}+69 z^{-4}+\cdots \\
& \kappa^{8}=z^{4}+4 z^{3}+6 z^{2}+8 z+9+20 z^{-1}+18 z^{-2}+92 z^{-3}+36 z^{-4}+\cdots
\end{aligned}
$$

\section{Hard hexagons}

The hard hexagon model can be realized as a lattice gas on a square lattice with an additional interaction along one diagonal $[1,16]$

$$
T_{1+t_{1}+2 t_{2}+\cdots+2^{m-1} t_{m}, 1+t_{1}+2 t_{2}^{\prime}+\cdots+2^{m-1} t_{m}^{\prime}}=\left(\prod_{j=1}^{m}\left(1-t_{j} t_{j+1}\right)\left(1-t_{j}^{\prime} t_{j+1}^{\prime}\right)\left(1-t_{j} t_{j}^{\prime}\right)\left(1-t_{j} t_{j+1}^{\prime}\right)\right) \sum^{\sum_{j=1}^{m}\left(t_{j}+t_{j}^{\prime}\right)}
$$

This matrix is considerably sparser than the hard squares transfer matrix, with many more null vectors. We find that for low fugacity $z<<1, m \times N$ periodic boundary conditions (tubes) result in

$$
\begin{aligned}
& Z_{4 \times N, \text {,ube }}^{I, N}=1+4 z-6 z^{2}+32 z^{3}-212 z^{4}+1568 z^{5}-12400 z^{6}+102592 z^{7}-876944 z^{8}+7683456 z^{9}+\cdots \\
& \kappa=Z^{\frac{1}{4}}=1+z-3 z^{2}+16 z^{3}-\frac{423}{4} z^{4}+\frac{3125}{4} z^{5}-\frac{24707}{4} z^{6}+51115 z^{7}-\frac{13987571}{32} z^{8}+\frac{122615141}{32} z^{9}+\cdots \\
& Z_{6 \times N, \text {,ube }}^{I N}=1+6 z-3 z^{2}+26 z^{3}-186 z^{4}+1440 z^{5}-11836 z^{6}+101586 z^{7}-900342 z^{8}+8178268 z^{9}+\cdots \\
& \kappa=Z^{\frac{1}{6}}=1+z-3 z^{2}+16 z^{3}-106 z^{4}+789 z^{5}-\frac{37907}{6} z^{6}+\frac{319129}{6} z^{7}-464388 z^{9}+\frac{25008499}{6} z^{9}+\cdots \\
& Z_{8 \times N, \text { athe }}^{1 / N}=1+8 z+4 z^{2}+16 z^{3}-134 z^{4}+1104 z^{5}-9384 z^{6}+82312 z^{7}-741276 z^{8}+6821040 z^{9}+\cdots \\
& \kappa=Z^{\frac{1}{8}}=1+z-3 z^{2}+16 z^{3}-106 z^{4}+789 z^{5}-6318 z^{6}+53198 z^{7}-\frac{7317383}{8} z^{8}+\frac{33392609}{8} z^{9}+\cdots
\end{aligned}
$$

These agree exactly up to and including the $z^{m-1}$ term with the known low-fugacity series expansions for the $N \times N$ model with $N \rightarrow \infty$, we begin to see finite size deviations beyond that point. We compare with the corner transfer matrix computation in the Kramers-Wannier approximation [16]:

$$
\begin{aligned}
& \sum_{b} F(a, b) A^{2}(b) F(b, a)=\xi A^{4}(a) \\
& \sum_{c} w(a, b, c) F(a, c) A(c) F(c, b)=\eta A(a) F(a, b) A(b) \\
& w(a, b, c)=z^{\frac{a+b+c}{6}} \varepsilon(a, b) \varepsilon(b, c) \varepsilon(c, a), \quad \kappa=\frac{\eta^{2}}{\xi}
\end{aligned}
$$

in which $\varepsilon(a, b)=1-a b, a, b \in\{0,1\}$. Truncating to $1 \times 1$ matrices we get

$$
\xi A^{4}(0)=A^{2}(1) F(0,1) F(1,0)+A^{2}(0) F^{2}(0,0)
$$




$$
\begin{aligned}
& \xi A^{4}(1)=A^{2}(0) F(0,1) F(1,0)+A^{2}(1) F^{2}(1,1) \\
& \eta A(0) F(0,0) A(0)=z^{\frac{1}{6}} A(1) F(0,1) F(1,0)+A(0) F^{2}(0,0) \\
& \eta A(0) F(0,1) A(1)=z^{\frac{1}{6}} A(0) F(0,1) F(0,0) \\
& \eta A(1) F(1,0) A(0)=z^{\frac{1}{6}} A(0) F(1,0) F(0,0) \\
& \eta A(1) F(1,1) A(1)=0
\end{aligned}
$$

from which we deduce

$$
F(1,1)=0, \quad F(0,1)=F(1,0)
$$

Set $A(0)=1, A(1)=A, F(0,1)=f, F(0,0)=g$, the corner transfer matrix equations define a one-dimensional affine variety in $(A, f, g, \xi, \kappa, z)$ space [17]

$$
\begin{aligned}
& \xi=A^{2} f^{2}+g^{2} \\
& \xi A^{4}=f^{2} \\
& \eta A=z^{\frac{1}{6}} g \\
& \eta g=z^{\frac{1}{6}} A f^{2}+g^{2}
\end{aligned}
$$

Eliminating $f, g$ and $\xi$ we obtain the elimination ideal generated by

$$
z^{\frac{1}{6}} A^{6}-\left(1-A^{6}\right)\left(z^{\frac{1}{6}}-A\right)=0, \quad \kappa=\frac{\left(1-A^{6}\right) z^{\frac{1}{3}}}{A^{2}}
$$

Expand the first of these in a Puiseux series by the Newton polygon method, and use the second to obtain $\kappa$ :

$$
\begin{aligned}
& A=z^{\frac{1}{6}}\left(1-z+5 z^{2}-34 z^{3}+265 z^{4}-2232 z^{5}+19766 z^{6}-181300 z^{7}+1706737 z^{8}+\cdots\right) \\
& \kappa=1+z-3 z^{2}+16 z^{3}-106 z^{4}+789 z^{5}-6319 z^{6}+53228 z^{7}-465258 z^{8}+\cdots
\end{aligned}
$$

a result in excellent agreement [16] with series computations produced in the 60's and 70's. The Newton polygon method is very well-suited to the study of corner transfer matrices if one can establish some of the elimination ideals of the variety defined by the corner transfer matrix equations. We include this example because the first of Eq. 49 is a true Puiseux series, and are an approximation for the $N \rightarrow \infty$ limit with which to compare Eq. 43.

Returning to the row-to-row transfer matrix, we can produce highfugacity expansions valid for $z>1$ :

$$
Z_{4 \times N}^{1 / N}=z+4-20 z^{-1}+236 z^{-2}-3468 z^{-3}+57044 z^{-4}-1005124 z^{-5}+18552156 z^{-6}-354084764 z^{-7}+\cdots
$$

$$
\begin{aligned}
& Z_{6 \times N}^{1 / N}=z^{2}+2 z+15+62 z^{-1}-92 z^{-2}-3392 z^{-3}-17462 z^{-4}+95678 z^{-5}+1919540 z^{-6}+7151412 z^{-7}+\cdots \\
& Z_{8 \times N}^{1 / N}=z^{\frac{5}{2}}+z^{2}+4 z^{\frac{3}{2}}+7 z+21 z^{\frac{1}{2}}-5-\frac{65}{2} z^{-\frac{1}{2}}-325 z^{-1}-\frac{593}{2} z^{-32}+919 z^{-2}+6221 z^{-\frac{5}{2}}+\cdots
\end{aligned}
$$

We also examined a variation (perhaps unstudied) on the hard hexagon model with Boltzmann weights for a face $w(a, b, c)=z^{(a+b+c) / 6}(1-a b c)$, and found excellent agreement between the $6 \times N$ tube and truncated corner transfer matricies for the infinite lattice

$$
\begin{aligned}
& \kappa_{6 \times N}=\left(1+z-2 z^{3}+7 z^{4}-12 z^{5}-15 z^{6}+194 z^{7}+\cdots\right) \\
& \kappa_{T C T M}=\left(1+z-2 z^{3}+7 z^{4}-12 z^{5}-15 z^{6}+193 z^{7}+\cdots\right)
\end{aligned}
$$

\section{Application to $m \times N$ Lattice Ising Models}

Ising $m \times N$ ribbons and tubes have transfer matrices with all matrix elements non-zero, and one finds that eigenvalues occur in approximate \pm -pairs, a spectral feature revealed by the first level Newton polygon segments, with very large gaps between largest and second largest eigenvalues of the same sign.

Ising ribbons on $m \times N$ lattices and tubes on $2 m \times N$ lattices have transfer matrices with the property

$$
P \cdot T(z) \cdot P^{\prime}=T\left(z^{-1}\right), \quad z=e^{-\beta J}
$$

in which $P, P^{\prime}$ are permutation (orthogonal) matrices with additional properties

$$
\left[P, P^{\prime}\right]=0, \quad P \cdot P^{\prime}=S, \quad[T(z), S]=0
$$

Consider a $3 \times N$ ribbon (Figure 5) of magnetic dipoles $S_{i}$ each of which can be in two states $s_{i}= \pm 1$, with magnetic dipole-dipole interactions between neighboring spins only.

The transfer matrix for this ribbon is $8 \times 8$ since each row of three spins has $2^{3}$ states, and the states of two adjacent rows label the rows and columns of the transfer matrix;

$$
\begin{aligned}
& T(z)_{\left(s_{1}, s_{2}, s_{3}\right),\left(s_{1}^{\prime}, s_{2}, s_{3}^{\prime}\right)}=e^{\left.-\beta J J\left(s_{1} s_{2}+s_{2} s_{3}+s_{1}^{\prime} s_{2}+s_{2} s_{3}^{\prime}\right) / 2+s_{1} s_{1}^{\prime}+s_{2} s_{2}+s_{3} s_{3}^{\prime}\right)}, \quad \beta=\frac{1}{k T}, \quad T \geq 0 \\
& =z^{\left(\left(s_{1} s_{2}+s_{2} s_{3}+s_{1}^{\prime} s_{2}^{\prime}+s_{2}^{\prime} s_{3}^{\prime}\right) / 2+s_{1} s_{1}^{\prime}+s_{2} s_{2}^{\prime}+s_{3} s_{3}^{\prime}\right)}, \quad z=e^{-\beta J}
\end{aligned}
$$

If $J<0 \quad(0<z<1)$ the model is ferromagnetic, otherwise antiferromagnetic. Note that the matrix is real and symmetric, and has symmetry under flipping all spins

$$
\begin{aligned}
& T(z)_{\left(s_{1}, s_{2}, s_{3}\right),\left(s_{1}^{\prime}, s_{2}^{\prime}, s_{3}^{\prime}\right)}=T(z)_{\left(-s_{1},-s_{2},-s_{3}\right),\left(-s_{1}^{\prime},-s_{2}^{\prime},-s_{3}^{\prime}\right)} \\
& T(z)_{\left(s_{1}, s_{2}, s_{3}\right),\left(s_{1}^{\prime}, s_{2}^{\prime}, s_{3}^{\prime}\right)}=T(z)_{\left(s_{1}^{\prime}, s_{2}^{\prime}, s_{3}^{\prime}\right),\left(s_{1}, s_{2}, s_{3}\right)}
\end{aligned}
$$

The inversion property Eq. 52 is due to the exponent being negated by flipping alternating spins in adjacent rows according to the pattern (for example again the $3 \times N$ case)

$$
T(z)_{\left(s_{1}, s_{2}, s_{3}\right),\left(s_{1}^{\prime}, s_{2}^{\prime}, s_{3}^{\prime}\right)}=T\left(z^{-1}\right)_{\left(s_{1},-s_{2}, s_{3}\right),\left(-s_{1}^{\prime}, s_{2}^{\prime},-s_{3}^{\prime}\right)}
$$

\section{The matrices $P, P^{\prime}$, and $S$}

Switch to a "bit-ordered" presentation of the states of each row of
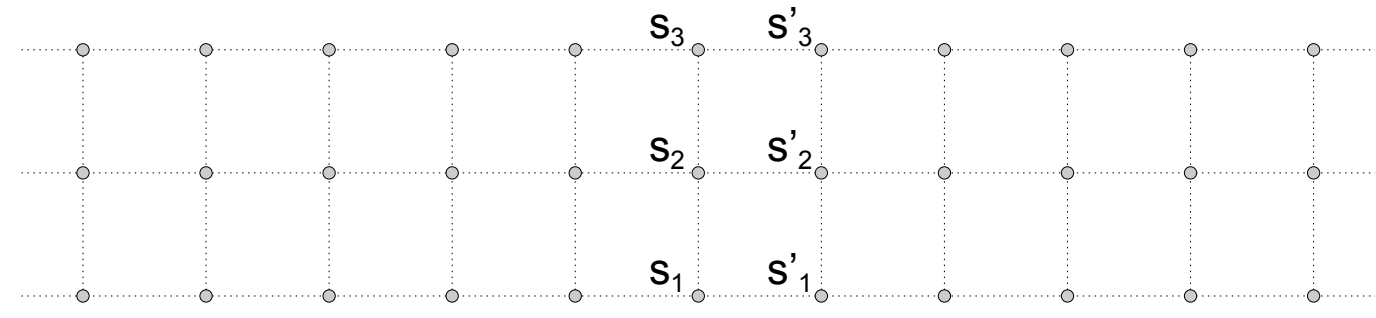

Figure 5: A section of the $3 \times N$ Ising ribbon. 
the lattice, let

$$
t_{i}=\left\{\begin{array}{lll}
0 & \text { if } & s_{i}=-1 \\
1 & \text { if } & s_{i}=1
\end{array}, \quad s_{i}=2 t_{i}-1\right.
$$

and label the rows and columns of the transfer matrix accordingly in binary, for the $3 \times N$ example

$$
T(z)_{\left(s_{1}, s_{2}, s_{3}\right),\left(s_{1}^{\prime}, s_{2}, s_{3}^{\prime}\right)}=T(z)_{1+t_{1}+2 t_{2}+4 t_{3}, 1+t_{1}+2 t_{2}^{\prime}+4 t_{3}^{\prime}}=T(z)_{i, j}, \quad i, j=1,2, \cdots, 8
$$

Define

$$
\overline{t_{i}}=\left\{\begin{array}{lll}
1 & \text { if } & t_{i}=0 \\
0 & \text { if } & t_{i}=1
\end{array}\right.
$$

then symmetry Eq. 52 can be expressed as

$$
T(z)_{1+t_{1}+2 t_{2}+4 t_{3}, 1+t_{1}^{\prime}+2 t_{2}^{\prime}+4 t_{3}^{\prime}}=T\left(z^{-1}\right)_{1+t_{1}+2 \bar{t}_{2}+4 t_{3}, 1+\bar{t}_{1}+2 t_{2}^{\prime}+4 \bar{t}_{3}}
$$

Let $P$ and $P$ ' be stochastic (permutation) matrices that are zeroes everywhere except

$$
P_{1+t_{1}+2 t_{2}+4 t_{3}, 1+t_{1}+2 \bar{t}_{2}+4 t_{3}}=1, \quad P_{1+t_{1}+2 t_{2}+4 t_{3}, 1+\bar{t}_{1}+2 t_{2}^{\prime}+4 \bar{t}_{3}}^{\prime}=1
$$

Note that $\left[P, P^{\prime}\right]=0$, and $P . P^{\prime}=S$ is the matrix with all ones down the upper right to lower left diagonal. The significance is that the transformation $z \rightarrow z^{-1}$ is a linear transformation of the transfer matrix

$$
T\left(z^{-1}\right)=P \cdot T(z) \cdot P^{\prime}
$$

and from the form of $P$ and $P^{\prime}$ one can see that

$$
\left[P, P^{\prime}\right]=0, \quad\left(P \cdot P^{\prime}\right)^{2}=1=S^{2}
$$

and the spin-negation symmetry Eq. 55 is simply

$$
S \cdot T(z) \cdot S=T(z)
$$

\section{Proof of the inversion property of largest eigenvalue}

Let $T(z)$ be a symmetric matrix $\left(2^{n} \times 2^{n}\right)$ all of whose matrix elements are of the form $z^{q}$ where $q$ is a finite rational number, and $z$ is a non-negative real variable.

The matrix $T(z)$ has the property that there exists two stochastic matrices $P, P^{\prime}$ (each row/column are unit vectors with only a single component non-zero, they are permutation matrices)

$$
\begin{aligned}
& P \cdot T(z) \cdot P^{\prime}=T\left(z^{-1}\right) \\
& {\left[P, P^{\prime}\right]=0, \quad P \cdot P^{\prime}=S, \quad S^{2}=1, \quad[T(z), S]=0}
\end{aligned}
$$

however $T(z)$ and $T\left(z^{-1}\right)$ are not similar. Then it is easy to show that

$$
\left(T\left(z^{-1}\right)\right)^{N}=P \cdot(T(z))^{N} \cdot P^{\prime}
$$

for any odd integer $N$.

$$
\begin{aligned}
& \left(T\left(z^{-1}\right)\right)^{N}=\left(P \cdot T(z) \cdot P^{\prime}\right)\left(P \cdot T(z) \cdot P^{\prime}\right) \cdots\left(P \cdot T(z) \cdot P^{\prime}\right)\left(P \cdot T(z) \cdot P^{\prime}\right) \\
& =P \cdot T(z) \cdot\left(P^{\prime} \cdot P\right) \cdot T(z) \cdot\left(P^{\prime} \cdot P\right) \cdots\left(P^{\prime} \cdot P\right) \cdot T(z) \cdot\left(P^{\prime} \cdot P\right) \cdot T(z) \cdot P^{\prime} \\
& =P \cdot T(z) \cdot(S) \cdot T(z) \cdot(S) \cdots(S) \cdot T(z) \cdot(S) \cdot T(z) \cdot P^{\prime} \\
& =P \cdot(S)^{N-1} \cdot(T(z))^{N} \cdot P^{\prime} \\
& =P \cdot(T(z))^{N} \cdot P^{\prime}
\end{aligned}
$$

For any vector $\mathbf{v}$ we have

$$
|P \cdot \mathbf{v}|=\left|P^{\prime} \cdot \mathbf{v}\right|=|\mathbf{v}|
$$

Let $\mathbf{V}$ be a vector of length one, all of whose components are positive. There are numbers $\left\{a_{i}, \mid i=1,2, \cdots, 2^{N}\right\}$ and $\left\{b_{i}, \mid i=1,2, \cdots, 2^{N}\right\}(T(z)$ and $T\left(z^{-1}\right)$ are Hermitian) such that

$$
P^{\prime} \cdot \mathbf{v}=\sum_{i} a_{i} \mathbf{u}_{i}, \quad \mathbf{v}=\sum_{i} b_{i} \mathbf{w}_{i}
$$

where $\left\{\bar{x}_{i}(z), \mathbf{u}_{i}\right\}$ are the ordered eigenvalue/eigenvector pairs of $T(z)$ and $\left\{\bar{y}_{i}(z), \mathbf{w}_{i}\right\}$ are the ordered eigenvalue/eigenvector pairs of $T\left(z^{-1}\right)$

$$
\bar{x}_{i} \geq \bar{x}_{i+1}, \quad \bar{y}_{i} \geq \bar{y}_{i+1}
$$

By the Frobenius-Perron theorem the largest eigenvalues $\bar{x}_{1}$ and $\bar{y}_{1}$ are non-degenerate, positive and correspond to eigenvectors with non-negative components.

$$
\left|\left(T\left(z^{-1}\right)\right)^{N} \cdot \mathbf{v}\right|=\left|P \cdot(T(z))^{N} \cdot P^{\prime} \cdot \mathbf{v}\right|, \quad\left|\sum_{i} b_{i} \bar{y}_{i}^{N} \mathbf{w}_{i}\right|=\left|P \cdot \sum_{i} a_{i} \bar{x}_{i}^{N} \mathbf{u}_{i}\right|
$$

As $N$ becomes very large, the two sides become

$$
\bar{y}_{1}^{N}\left|b_{1} \mathbf{w}_{1}\right|+\mathcal{O}\left(\frac{\bar{y}_{2}}{\bar{y}_{1}}\right)^{N}=\bar{y}_{1}^{N} b_{1}+\mathcal{O}\left(\frac{\bar{y}_{2}}{\bar{y}_{1}}\right)^{N}=\bar{x}_{1}^{N}\left|a_{1} P \cdot \mathbf{u}_{1}\right|+\mathcal{O}\left(\frac{\bar{x}_{2}}{\bar{x}_{1}}\right)^{N}=\bar{x}_{1}^{N} a_{1}+\mathcal{O}\left(\frac{\bar{x}_{2}}{\bar{x}_{1}}\right)^{N}
$$

and therefore for any $z$, by dividing these equations for consecutive odd integers $N$ and $N+2$ as $N \rightarrow \infty$

$$
\bar{x}_{1}^{2}=\bar{y}_{1}^{2}, \quad \bar{x}_{1}=\bar{y}_{1}
$$

the phases being set to unity by the Frobenius-Perron theorem.

Even more can be said about the spectra of $T(z)$ and $T\left(z^{-1}\right)$ (ferromagnetic/anti-ferromagnetic Ising models). It is easy to show that

$\bar{y}_{i}= \pm \bar{x}_{i}, \quad i>1$

Since $\left[T\left(z^{-1}\right), S\right]=0$ there is a unitary matrix $U$ such that

$$
U \cdot T\left(z^{-1}\right) \cdot U^{-1}=\Delta(z), \quad U \cdot S \cdot U^{-1}=\Sigma
$$

in which $\Delta(z)=\operatorname{diag}\left(\bar{y}_{1}, \bar{y}_{2}, \cdots\right)$ and $\Sigma$ is diagonal with half of its diagonal entries being 1 , the other half being -1 . Then since $P$ is orthogonal, $P \cdot T(z) \cdot P^{-1}$ and $T(z)$ are similar

$$
\begin{aligned}
& P \cdot T(z) \cdot P^{\prime}=T\left(z^{-1}\right)(77) \\
& P \cdot T(z) \cdot\left(P^{-1} \cdot P\right) \cdot P^{\prime}=P \cdot T(z) \cdot P^{-1} \cdot S \\
& P \cdot T(z) \cdot P^{-1}=T\left(z^{-1}\right) \cdot S \\
& U \cdot\left(P \cdot T(z) \cdot P^{-1}\right) \cdot U^{-1}=U \cdot T\left(z^{-1}\right) \cdot S \cdot U^{-1} \\
& U \cdot\left(P \cdot T(z) \cdot P^{-1}\right) \cdot U^{-1}=\left(U \cdot T\left(z^{-1}\right) \cdot U^{-1}\right) \cdot\left(U \cdot S \cdot U^{-1}\right) \\
& (U \cdot P) \cdot T(z) \cdot(U \cdot P)^{-1}=\Delta(z) \cdot \Sigma
\end{aligned}
$$

The right side of this expression is a diagonal matrix, therefore the left side must be as well, call it $\Lambda(z)=\operatorname{diag}\left(\bar{x}_{i_{1}}, \bar{x}_{i_{2}}, \cdots\right)$

$\Lambda(z)=\Delta(z) \cdot \Sigma$

An interesting bit of algebraic geometry is that models whose transfer matrix $T(z)$ has property Eq. 52 have characteristic polynomials $P(x, z)=\operatorname{det}(T(z)-x)$ which have Newton diagrams with convex hulls possessing a reflection symmetry about a horizontal line Figure 6.

\section{Results for the Ising ribbons and tubes}

The ribbons possess the inversion property for all $m$, but the tubes only for $m$ even (and recall that $z=e^{-\beta J}$ ).

$Z_{3 \times N, r i b}^{1 / N}=z^{-5}+3 z+7 z^{3}+12 z^{5}+18 z^{7}+13 z^{9}-41 z^{11}-222 z^{13}-648 z^{15}-1295 z^{17}+\cdots \quad z<<1 \quad$ (79) $Z_{3 \times N, r i b}^{1 / N}=z^{5}+3 z^{-1}+7 z^{-3}+12 z^{-5}+18 z^{-7}+13 z^{-9}-41 z^{-11}-222 z^{-13}-648 z^{-15}-1295 z^{-17} \ldots \quad z \gg>1$ 
Ising $2 \times \mathrm{N}$

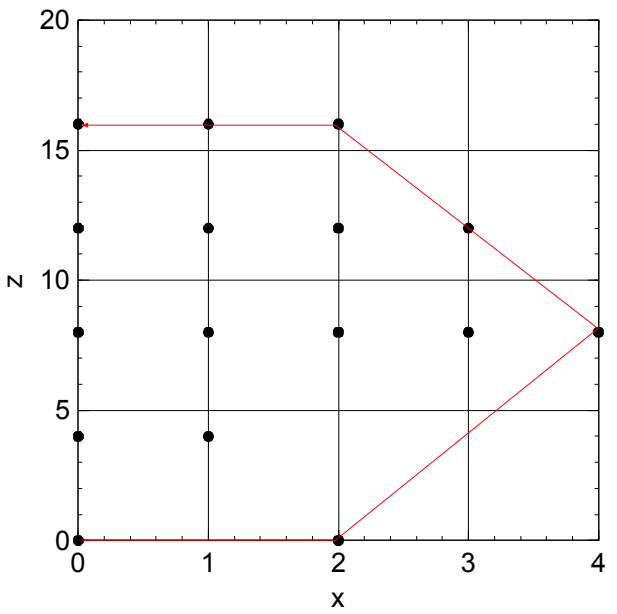

Hard squares $2 \times \mathrm{N}$

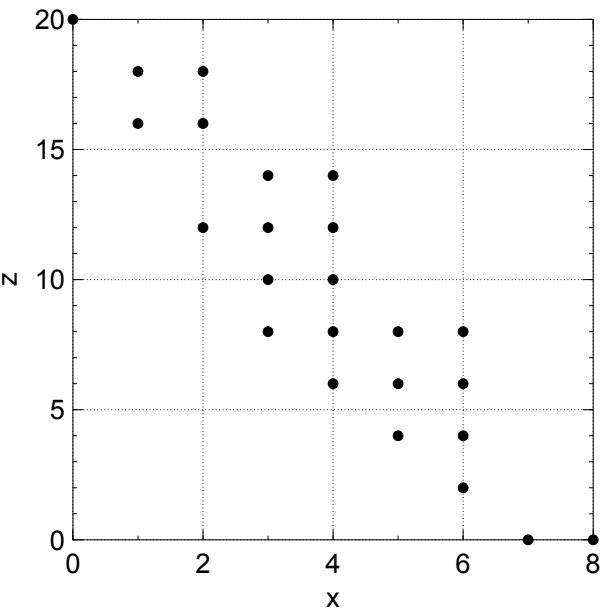

Figure 6: A comparison of models with Newton diagram hulls with and without the reflection symmetry.

$Z_{3 \times N, \text { tube }}^{1 / N}=z^{-4}+2 z^{-2}+2+z^{2}+3 z^{4}-3 z^{10}-3 z^{12}+12 z^{14}-18 z^{16}+45 z^{18}-69 z^{20} \cdots \quad z<<1$

$Z_{3 \times N, \text { tube }}^{1 / N}=z^{6}+1+3 z^{-2}+6 z^{-4}+6 z^{-6}+9 z^{-8}+9 z^{-10}-6 z^{-12}+36 z^{-14}-99 z^{-16}-213 z^{-18}+\cdots \quad z>>1$

\begin{tabular}{|c|c|c|c|c|}
\hline \multirow[b]{2}{*}{$z$} & \multicolumn{2}{|c|}{ Ising $3 \times N$ ribbon } & \multicolumn{2}{|c|}{ Ising $3 \times N$ tube } \\
\hline & $\bar{x}_{\max }$ (octave) & $\bar{x}_{\max }$ (Eq. 79) & $\bar{x}_{\max }$ (octave) & $\bar{x}_{\max }$ (Eq. 79) \\
\hline 0.05 & $\begin{array}{c}3.20000015087876 \\
E+06\end{array}$ & $\begin{array}{c}3.20000015087876 \\
E+06\end{array}$ & $\begin{array}{c}1.60802002518750 \\
E+05\end{array}$ & $\begin{array}{c}1.60802002518750 \\
E+05\end{array}$ \\
\hline 0.1 & $\begin{array}{c}1.0000030712181 \\
3 E+05\end{array}$ & $\begin{array}{c}1.0000030712181 \\
2 \mathrm{E}+05\end{array}$ & $\begin{array}{c}1.02020102999997 \\
E+04\end{array}$ & $\begin{array}{c}1.02020102999997 \\
E+04\end{array}$ \\
\hline 0.2 & $\begin{array}{c}3.125660076011 \\
46 E+03\end{array}$ & $\begin{array}{l}3.125660076011 \\
53 E+03\end{array}$ & $\begin{array}{c}6.77044799682371 \\
E+02\end{array}$ & $\begin{array}{c}6.77044799682371 \\
E+02\end{array}$ \\
\hline 0.25 & $\begin{array}{c}1.02487222820783 \\
E+03\end{array}$ & $\begin{array}{c}1.0248722282 \\
E+03\end{array}$ & $\begin{array}{c}2.900742157512 \\
70 \mathrm{E}+02\end{array}$ & $\begin{array}{c}2.900742157512 \\
68 \mathrm{E}+02\end{array}$ \\
\hline 0.3 & $\begin{array}{c}4.12644867125248 \\
\text { E+02 }\end{array}$ & $\begin{array}{c}4.12644867229159 \\
E+02\end{array}$ & $\begin{array}{c}1.47793293548279 \\
E+02\end{array}$ & $\begin{array}{c}1.47793293548156 \\
E+02\end{array}$ \\
\hline 4 & $\begin{array}{c}1.02487222820783 \\
E+03\end{array}$ & $\begin{array}{c}1.0248722282 \\
E+03\end{array}$ & $\begin{array}{c}4.09721254773772 \\
E+03\end{array}$ & $\begin{array}{c}4.09721254800625 \\
E+03\end{array}$ \\
\hline 10 & $\begin{array}{c}1.00000307121813 \\
E+05\end{array}$ & $\begin{array}{c}1.00000307121813 \\
E+05\end{array}$ & $\begin{array}{c}1.00000103060609 \\
E+06\end{array}$ & $\begin{array}{c}1.00000103060609 \\
E+06\end{array}$ \\
\hline 20 & $\begin{array}{c}3.20000015087876 \\
E+06\end{array}$ & $\begin{array}{c}3.20000015087876 \\
E+06\end{array}$ & $\begin{array}{c}6.40000010075376 \\
E+07\end{array}$ & $\begin{array}{c}6.40000010075376 \\
E+07\end{array}$ \\
\hline
\end{tabular}

$Z_{4 \times N, r i b}^{1 / N}=z^{-7}+2 z^{-1}+5 z+10 z^{3}+28 z^{5}+54 z^{7}+84 z^{9}+86 z^{11}-56 z^{13}-698 z^{15}+\cdots \quad z<<1 \quad(80)$

$Z_{4 \times N, r i b}^{1 / N}=z^{7}+2 z+5 z^{-1}+10 z^{-3}+28 z^{-5}+54 z^{-7}+84 z^{-9}+86 z^{-11}-56 z^{-13}-698 z^{-15}+\cdots \quad z \gg>1$

$Z_{4 \times N, \text { tube }}^{1 / N}=z^{-8}+5+20 z^{4}+64 z^{8}+116 z^{12}-140 z^{16}+2556 z^{20}+\cdots \quad z<<1$

$Z_{4 \times N, \text { tube }}^{1 / N}=z^{8}+5+20 z^{-4}+64 z^{-8}+116 z^{-12}-140 z^{-16}+2556 z^{-20}+\cdots \quad z>>1$

\begin{tabular}{|c|c|c|c|c|}
\hline & \multicolumn{2}{|c|}{ Ising $4 \times N$ ribbon } & \multicolumn{2}{c|}{ Ising $4 \times N$ tube } \\
\hline$z$ & $\bar{x}_{\max }$ (octave) & $\bar{x}_{\max }$ (Eq. 80) & $\bar{x}_{\max }$ (octave) & $\bar{x}_{\max }$ (Eq. 80) \\
\hline 0.05 & $\begin{array}{c}1.28000004025 \\
\mathrm{e}+09\end{array}$ & $\begin{array}{c}1.28000004025 \\
\mathrm{e}+09\end{array}$ & $\begin{array}{c}2.56000000050001 \\
\mathrm{e}+10\end{array}$ & $\begin{array}{c}2.56000000050001 \\
\mathrm{e}+10\end{array}$ \\
\hline 0.1 & 1.00000205103 & 1.00000205103 & 1.00000005002001 & 1.00000005002001 \\
& $\mathrm{e}+07$ & $\mathrm{e}+07$ & $\mathrm{e}+08$ & $\mathrm{e}+08$ \\
\hline 0.2 & 7.81360896959 & 7.81360896959 & 3.90630032164314 & 3.90630032164314 \\
& $\mathrm{e}+04$ & $\mathrm{e}+04$ & $\mathrm{e}+05$ & $\mathrm{e}+05$ \\
\hline 0.25 & 1.63934372289 & 1.63934372291 & 6.55410791084417 & 6.55410791084464 \\
& $\mathrm{e}+04$ & $\mathrm{e}+04$ & $\mathrm{e}+04$ & $\mathrm{e}+04$ \\
\hline
\end{tabular}

\begin{tabular}{|c|c|c|c|c|}
\hline 0.3 & $\begin{array}{c}4.58099200670 \\
\mathrm{e}+03\end{array}$ & $\begin{array}{c}4.58099201152 \\
\mathrm{e}+03\end{array}$ & $\begin{array}{c}1.52467452875790 \\
\mathrm{e}+04\end{array}$ & $\begin{array}{c}1.52467452877609 \\
\mathrm{e}+04\end{array}$ \\
\hline 4 & 1.63934372289 & 1.63934372291 & 6.55410791084417 & 6.55410791084464 \\
$\mathrm{e}+04$ & $\mathrm{e}+03$ & $\mathrm{e}+04$ & 04 \\
\hline 10 & 1.00000205102855 & 1.00000205103 & 1.00000005002001 & 1.00000005002001 \\
& $e+07$ & $e+07$ & $e+08$ & $e+08$ \\
\hline 20 & $\begin{array}{c}1.28000004025126 \\
\mathrm{e}+09\end{array}$ & $\begin{array}{c}1.28000004025 \\
\mathrm{e}+09\end{array}$ & $\begin{array}{c}2.56000000050001 \\
\mathrm{e}+10\end{array}$ & $\begin{array}{c}2.56000000050001 \\
\mathrm{e}+10\end{array}$ \\
\hline
\end{tabular}

$Z_{5 \times N, r i b}^{1 / N}=z^{-9}+2 z^{-3}+5 z^{-1}+5 z+20 z^{3}+52 z^{5}+129 z^{7}+245 z^{9}+\cdots \quad z<<1 \quad(81)$

$Z_{5 \times N, r i b}^{1 / N}=z^{9}+2 z^{3}+5 z^{1}+5 z^{-1}+20 z^{-3}+52 z^{-5}+129 z^{-7}+245 z^{-9}+\cdots \quad z>>1$

$Z_{5 \times N, \text { tube }}^{1 / N}=z^{-8}+2 z^{-6}+2 z^{-4}+2 z^{-2}+7+11 z^{2}+20 z^{4}+10 z^{6}-25 z^{8}+\cdots \quad z<<1$

$Z_{5 \times N, \text { tube }}^{1 / N}=z^{10}+5 z^{2}+1+10 z^{-2}+20 z^{-4}+35 z^{-6}+105 z^{-8}+110 z^{-10}+280 z^{-12}+\cdots \quad z>>1$

\begin{tabular}{|c|c|c|c|c|}
\hline \multirow[b]{2}{*}{$z$} & \multicolumn{2}{|c|}{ Ising $5 \times N$ ribbon } & \multicolumn{2}{|c|}{ Ising $5 \times N$ tube } \\
\hline & $\bar{x}_{\max }$ (octave) & $\bar{x}_{\max }($ Eq. 81) & $\bar{x}_{\max }$ (octave) & $\bar{x}_{\max }$ (Eq. 81) \\
\hline 0.05 & $\begin{array}{c}5.12000016100253 \\
e+11\end{array}$ & $\begin{array}{c}5.12000016100253 \\
e+11\end{array}$ & $\begin{array}{c}2.5728320807 \\
\mathrm{e}+10\end{array}$ & $\begin{array}{c}2.5728320807 \\
e+10\end{array}$ \\
\hline 0.1 & $\begin{array}{c}1.00000205052053 \\
\mathrm{e}+09\end{array}$ & $\begin{array}{c}1.00000205052053 \\
\mathrm{e}+09\end{array}$ & $\begin{array}{c}1.0202020711 \\
\mathrm{e}+08\end{array}$ & $\begin{array}{c}1.0202020711 \\
\mathrm{e}+08\end{array}$ \\
\hline 0.2 & $\begin{array}{c}1.95340117842540 \\
\mathrm{e}+06\end{array}$ & $\begin{array}{c}1.95340117841664 \\
\text { e+06 }\end{array}$ & $\begin{array}{c}4.2318247279 \\
e+05\end{array}$ & $\begin{array}{c}4.2318247258 \\
e+05\end{array}$ \\
\hline 0.25 & $\begin{array}{c}2.62293622193879 \\
\text { e+05 }\end{array}$ & $\begin{array}{c}2.62293622089386 \\
\text { e+05 }\end{array}$ & $\begin{array}{c}7.4279768402 \\
\mathrm{e}+04\end{array}$ & $\begin{array}{c}7.4279767685 \\
e+04\end{array}$ \\
\hline 0.3 & $\begin{array}{c}5.08982043587032 \\
e+04\end{array}$ & $\begin{array}{c}5.08982035606666 \\
\mathrm{e}+04\end{array}$ & $\begin{array}{c}1.8262359688 \\
e+04\end{array}$ & $\begin{array}{c}1.82623567048 \\
e+04\end{array}$ \\
\hline 4 & $\begin{array}{c}2.62293622193880 \\
e+05\end{array}$ & $\begin{array}{c}2.62293622089386 \\
e+05\end{array}$ & $\begin{array}{c}1.0486577134 \\
\mathrm{e}+06\end{array}$ & $\begin{array}{c}1.0486577133 \\
\mathrm{e}+06\end{array}$ \\
\hline 10 & $\begin{array}{c}1.00000205052053 \\
\mathrm{e}+09\end{array}$ & $\begin{array}{c}1.00000205052053 \\
\mathrm{e}+09\end{array}$ & $\begin{array}{c}1.0000000501 \\
e+10\end{array}$ & $\begin{array}{c}1.0000000501 \\
e+10\end{array}$ \\
\hline 20 & $\begin{array}{c}5.12000016100253 \\
\mathrm{e}+11\end{array}$ & $\begin{array}{c}5.12000016100253 \\
e+11\end{array}$ & $\begin{array}{c}1.0240000002 \\
\mathrm{e}+13\end{array}$ & $\begin{array}{c}1.0240000002 \\
e+13\end{array}$ \\
\hline
\end{tabular}

The precision of the series expansions become astounding at $m=6$;

$$
\begin{aligned}
& Z_{6 \times N, \text { tube }}^{1 / N}=z^{-12}+6 z^{-4}+13+75 z^{4}+378 z^{8}+1420 z^{12}+3933 z^{16}+\cdots \quad z<<1 \quad(82) \\
& Z_{6 \times N, \text { tube }}^{1 / N}=z^{12}+6 z^{4}+13+75 z^{-4}+378 z^{-8}+1420 z^{-12}+3933 z^{-16}+\cdots \quad z>>1
\end{aligned}
$$




\begin{tabular}{|l|l|l|}
\hline \multicolumn{2}{|l|}{ Ising $6 \times N$ tube } \\
\hline$z$ & $\bar{x}_{\max }$ (octave) & $\bar{x}_{\max }$ (Eq. 82) \\
\hline 0.05 & $4.09600000096002 \mathrm{e}+15$ & $4.09600000096001 \mathrm{e}+15$ \\
\hline 0.1 & $1.00000006001301 \mathrm{e}+12$ & $1.00000006001301 \mathrm{e}+12$ \\
\hline 0.2 & $2.44144388120973 \mathrm{e}+08$ & $2.44144388120973 \mathrm{e}+08$ \\
\hline 0.25 & $1.67787652988221 \mathrm{e}+07$ & $1.67785092988221 \mathrm{e}+07$ \\
\hline 0.3 & $1.88243079697202 \mathrm{e}+06$ & $1.88230734018170 \mathrm{e}+06$ \\
\hline 4 & $1.67787652988222 \mathrm{e}+07$ & $1.67787652988221 \mathrm{e}+07$ \\
\hline 10 & $1.00000006001301 \mathrm{e}+12$ & $1.00000006001301 \mathrm{e}+12$ \\
\hline 20 & $4.09600000096001 \mathrm{e}+15$ & $4.09600000096001 \mathrm{e}+15$ \\
\hline
\end{tabular}

We report the partition functions per particle $\kappa_{m \times N}=Z_{m \times N}^{1 m N}$

$\kappa_{3 \times N}=z^{2}+\frac{1}{3} z^{-4}+z^{-6}+2 z^{-8}+\frac{17}{9} z^{-10}+\frac{7}{3} z^{-12}+\frac{2}{3} z^{-14}+\cdots$

$\kappa_{4 \times N}=z^{2}+\frac{5}{4} z^{-6}+5 z^{-10}+\frac{437}{32} z^{-14}+\frac{41}{4} z^{-18}+\cdots$

$\kappa_{5 \times N}=z^{2}+z^{-6}+15 z^{-8}+2 z^{-10}+4 z^{-12}+5 z^{-14}+1015 z^{-16}+\cdots$

$\kappa_{6 \times N}=z^{2}+z^{-6}+\frac{13}{6} z^{-10}+10 z^{-14}+\frac{313}{6} z^{-18}+\cdots$

\section{Polynomial and degenerate eigenvalues}

We discover some interesting surprises scattered among the eigenvalues computed by these methods. Some Ising systems possess eigenvalues with truncated series expansion, polynomials. For the $3 \times N$ Ising tube we find that there are two pairs of degenerate eigenvalues whose series truncate after a few terms. The signal for this termination is that the Newton polygon construction gives no new segments.

$$
\begin{aligned}
& \bar{x}_{\text {max }}=\bar{x}_{1}=z^{6}+1+3 z^{-2}+6 z^{-4}+6 z^{-6}+9 z^{-8}+9 z^{-10}-6 z^{-12}-36 z^{-14}-99 z^{-16}+\cdots \\
& \bar{x}_{2}=z^{6}-1+3 z^{-2}-6 z^{-4}+6 z^{-6}-9 z^{-8}+9 z^{-10}+6 z^{-12}-36 z^{-14}+99 z^{-16}+\cdots \\
& \bar{x}_{3}=z^{2}+2-z^{-2}-5 z^{-4}-6 z^{-6}-9 z^{-8}-9 z^{-10}+6 z^{-12}+36 z^{-14}+99 u^{-16}+\cdots \\
& \bar{x}_{4,5}=z^{2}+1-z^{-2}-z^{-4} \\
& \bar{x}_{6,7}=z^{2}-1-z^{-2}+z^{-4} \\
& \bar{x}_{8}=z^{2}-2-z^{-2}+5 z^{-4}-6 z^{-6}+9 z^{-8}-9 z^{-10}-6 z^{-12}+36 z^{-14}-99 u^{-16}+\cdots
\end{aligned}
$$

For the $4 \times N$ Ising tube, $z \rightarrow u^{-1}$ we obtain three branches at the first level

$$
Q=\left(x u^{8}-1\right)^{2}, \quad x^{2}\left(x u^{4}-1\right)^{12}, \quad(x-1)^{2}
$$

indicating two (large) roots starting with $u^{-8}=z^{8}$, twelve starting with $u^{-4}=z^{4}$ and two starting with 1 .

Take the twelve-fold branch and substitute $x \rightarrow x+u^{-4}$, the new Newton polygon exhibits branches

$$
Q=\left(u^{2} x-2\right)^{2}\left(u^{2} x+2\right)^{2}\left(u^{4} x^{2}-8\right), \quad x-3 u^{4}, \quad Q=x(x+4)(x+2)^{4}
$$

Follow the branch $x \rightarrow x+u^{-4}$, it does not fork, there is only one new (degenerate) Newton polygon segment

$$
Q=\left(x+2 u^{2}\right)^{2}
$$

which we follow with $x \rightarrow x-2 u^{2}$, again it does not fork, there is only one new (degenerate) segment

$$
Q=\left(x+u^{4}\right)^{2}
$$

which terminates at the next level (no new segments are produced), giving the (double) polynomial root (far left branch set of Figure 7).

$$
\bar{x}=u^{-4}+2 u^{-2}-2 u^{2}-u^{4}=z^{4}+2 z^{2}-2 z^{-2}-z^{-4}
$$

Following another branch of the twelve-fold $x \rightarrow x+u^{-4} \rightarrow x-2 u^{-2}$ segment, namely

$$
Q=\left(x-2 u^{2}\right)^{2}
$$

another step to

$$
Q=\left(x+u^{4}\right)^{2}
$$

we come to another terminating line, giving the double root

$$
\bar{x}=u^{-4}-2 u^{-2}+2 u^{2}-u^{4}=z^{4}-2 z^{2}+2 z^{-2}-z^{-4}
$$

Return to second node, and follow the branch $x \rightarrow x+u^{-4} \rightarrow x-2$ to yet another fork

$$
Q=x^{2}-4, \quad Q=\left(x-u^{4}\right)^{4}
$$

the second branch of this fork promptly terminates on the quadruple root

$$
\bar{x}=u^{-4}-2+u^{4}=z^{4}-2+z^{-4}
$$

The significance of polynomial roots, and especially degenerate polynomial roots, is that they are rather easy to find by the Newton polygon method since they require few iterations. They appear to follow a clear pattern. Once they are found, they can be factored out of the characteristic polynomial exactly, reducing its order substantially. This not only simplifies calculation of the remaining, more significant roots, but makes it possible to tackle even larger systems.

This example contains the first case that we have illustrated in which a root has non-rational coefficients: look at the branch beginning with the factor $\left(u^{4} x^{2}-8\right)$. The best way to deal with such a case is by treating it symbolically [12]

\section{let ! $\mathrm{C}^{\wedge} 2-8=0$;}

and develop the root in a series in the two variables $C$ and $u$, obtaining in fact two roots (the third largest and third from smallest) since there are two solutions to $\quad 8=0$

$$
\begin{aligned}
& x=u^{-4}+8 C^{-1} u^{-2}+2-8 C^{-1} u^{2}-7 u^{4}-48 C^{-1} u^{6}-32 u^{8}-144 C^{-1} u^{10}-56 u^{12}-112 C^{-1} u^{14}+\cdots \\
& \bar{x}_{3}=z^{4}+\sqrt{8} z^{2}+2-\sqrt{8} z^{-2}-7 z^{-4}-6 \sqrt{8} z^{-6}-32 z^{-8}-18 \sqrt{8} z^{-10}-56 z^{-12}-14 \sqrt{8} z^{-14}+\cdots \\
& \bar{x}_{14}=z^{4}-\sqrt{8} z^{2}+2+\sqrt{8} z^{-2}-7 z^{-4}+6 \sqrt{8} z^{-6}-32 z^{-8}+18 \sqrt{8} z^{-10}-56 z^{-12}+14 \sqrt{8} z^{-14}+\cdots
\end{aligned}
$$

the third largest eigenvalue of the transfer matrix.

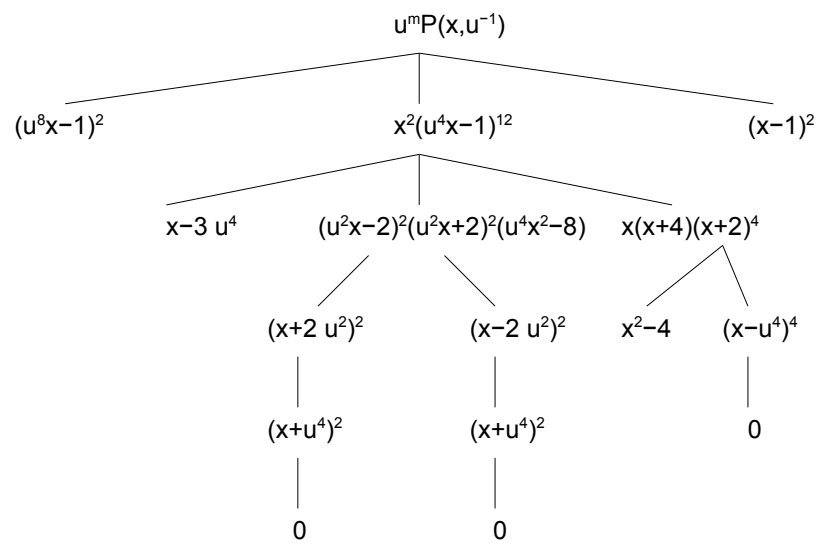

Figure 7: Illustration of the lineage ( $Q$-polynomials) of degenerate polynomial eigenvalues for the $4 \times N$ Ising tube. 
The full set of eigenvalue expansions for the $4 \times N$ ferromagnetic Ising tube are as follows:

$$
\begin{aligned}
& \bar{x}_{1}=z^{8}+5+20 z^{-4}+64 z^{-8}+116 z^{-12}-140 z^{-16}+2556 z^{-20}+\cdots \\
& \bar{x}_{2}=z^{8}+3-4 z^{-4}-12 z^{-8}+12 z^{-12}+84 z^{-16}-84 z^{-20}+\cdots \\
& \bar{x}_{3}=z^{4}+\sqrt{8} z^{2}+2-\sqrt{8} z^{-2}-7 z^{-4}-6 \sqrt{8} z^{-6}-32 z^{-8}-18 \sqrt{8} z^{-10}-56 z^{-12}-14 \sqrt{8} z^{-14}+64 z^{-16}+\cdots \\
& \bar{x}_{4,5}=z^{4}+2 z^{2}-2 z^{-2}-z^{-4} \\
& \bar{x}_{6}=z^{4}+3 z^{-4}-4 z^{-8}-12 z^{-12}+12 z^{-16}+84 z^{-20}+\cdots \\
& \bar{x}_{7,8,9,10}=z^{4}-2+z^{-4} \\
& \bar{x}_{11}=z^{4}-4+3 z^{-4}+12 z^{-8}-12 z^{-12}-84 z^{-16}+84 z^{-20}+\cdots \\
& \bar{x}_{12,13}=z^{4}-2 z^{2}+2 z^{-2}-z^{-4} \\
& \bar{x}_{14}=z^{4}-\sqrt{8} z^{2}+2+\sqrt{8} z^{-2}-7 z^{-4}+6 \sqrt{8} z^{-6}-32 z^{-8}+18 \sqrt{8} z^{-10}-56 z^{-12}+14 \sqrt{8} z^{-14}+64 z^{-16}+\cdots \\
& \bar{x}_{15}=1-4 z^{-4}+3 z^{-8}+12 z^{-12}-12 z^{-16}-84 z^{-20}+\cdots \\
& \bar{x}_{16}=1-4 z^{-4}+z^{-8}-4 z^{-12}+12 z^{-16}+140 z^{-20}+560 z^{-24}+\cdots
\end{aligned}
$$

The $4 \times N$ hard-squares model on a tube (low fugacity) also has polynomial (in fact monomial) eigenvalues, as well as a large null-space

$$
\begin{aligned}
& \bar{x}_{1}=1+4 z-2 z^{2}+12 z^{3}-62 z^{4}+352 z^{5}-2122 z^{6}+13344 z^{7}-86566 z^{8}+\cdots \quad z<<1 \quad(97) \\
& \bar{x}_{2}=z^{2}-2 z^{3}+4 z^{4}-6 z^{5}+50 z^{7}-268 z^{8}+990 z^{9}+\cdots \\
& \bar{x}_{3}=z^{2}-2 z^{3}+6 z^{4}-22 z^{5}+90 z^{6}-394 z^{7}+1806 z^{8}-8558 z^{9}+\cdots \\
& \bar{x}_{4-12}=0 \\
& \bar{x}_{13}=-z+2 z^{2}-10 z^{3}+58 z^{4}-346 z^{5}+2122 z^{6}-13394 z^{7}+86834 z^{8}+\cdots \\
& \bar{x}_{14,15}=-z \\
& \bar{x}_{16}=-z-2 z^{2}+2 z^{3}-6 z^{4}+22 z^{5}-90 z^{6}+394 z^{7}-1806 z^{8}+\cdots
\end{aligned}
$$

and for high fugacity we also find polynomial (monomial) roots

$$
\begin{aligned}
& \bar{x}_{1}=z^{2}+2 z+4-2 z^{-1}-8 z^{-2}+22 z^{-3}-190 z^{-5}+120 z^{-6}+1442 z^{-7}-3268 z^{-8}, \quad z \gg>1 \quad(98) \\
& \bar{x}_{2}=z-2+6 z^{-1}-22 z^{-2}+90 z^{-3}-394 z^{-4}+1806 z^{-5}-8558 z^{-6}+41586 z^{-7}-206098 z^{-8}+\cdots \\
& \bar{x}_{3}=z-2+2 z^{-1}+6 z^{-2}-22 z^{-3}-10 z^{-4}+186 z^{-5}-178 z^{-6}-1390 z^{-7}+3630 z^{-8}+\cdots \\
& \bar{x}_{4 \rightarrow 12}=0 \\
& \bar{x}_{13}=-1+2 z^{-2}-10 z^{-4}+4 z^{-5}+58 z^{-6}-52 z^{-7}-362 z^{-8}+544 z^{-9}+\cdots \\
& \bar{x}_{14,15}=-z \\
& \bar{x}_{16}=-z^{2}-2 z+2-6 z^{-1}+22 z^{-2}-90 z^{-3}+394 z^{-4}-1806 z^{-5}+8558 z^{-6}-41586 z^{-7}+206098 z^{-8}+\cdots
\end{aligned}
$$

Correlation functions and other interesting properties can be computed if one has all of the eigenvalues of the transfer matrix. Let $U$ be a unitary matrix that diagonalizes $T(z)$, in other words let $U \cdot T(z) \cdot U^{-1}=\Lambda(z)=\operatorname{diag}\left(\bar{x}_{1}, \bar{x}_{2}, \cdots\right)$, then $\left(\bar{x}_{1}\right.$ is the largest eigenvalue $)$ with $\sigma=U \cdot s \cdot U^{-1}$

$$
\left\langle s_{j} s_{k}\right\rangle=\frac{\operatorname{Tr}\left(\sigma \cdot \Lambda^{N-(j-k)}(z) \cdot \sigma \cdot \Lambda^{j-k}\right)}{\operatorname{Tr}\left(\Lambda^{N}(z)\right)} \rightarrow \sum_{\ell \neq 1} a_{\ell}\left(\frac{\bar{x}_{\ell}}{\bar{x}_{1}}\right)^{j-k}, N \rightarrow \infty
$$

the coefficients $a$ being given by the various components of $U$. Hensel constructions can be used to construct the corresponding eigenvector components in Puiseux series for non-degenerate eigenvalues [18].

\section{The Baxter-Wu model}

The Baxter-Wu model, with Hamiltonian

$$
H=-J \sum_{\text {faces } i} s_{i, 1} s_{i, 2} s_{i, 3}, \quad s_{i, n} \in\{-1,1\}
$$

in which we sum over all faces of the (triangular) lattice, each face having three vertices (with states $S_{i, n}, n=1,2,3$ ) provides us with an example in which second-largest magnitude eigenvalues are complex. For the $4 \times N$ tube we find

$\bar{x}_{\max }=z^{-8}+4 z^{4}+13 z^{8}+64 z^{12}+356 z^{16}+1980 z^{20}+10792 z^{24}+\cdots, \quad z=e^{\beta,}$

\begin{tabular}{|l|l|l|}
\hline \multicolumn{2}{|l|}{ Baxter-Wu $4 \times N$ tube } \\
\hline$z$ & Octave & Series \\
\hline 0.1 & $1.00000000000400 \mathrm{e}+08$ & $1.00000000000 \mathrm{e}+0.8$ \\
\hline 0.2 & $3.90625006433545 \mathrm{e}+05$ & $3.90625006434 \mathrm{e}+5$ \\
\hline 0.3 & $1.52416123161342 \mathrm{e}+04$ & 15241.6123161 \\
\hline 0.4 & $1.52599107786097 \mathrm{e}+03$ & 1525.99107738 \\
\hline 0.5 & 256.324688942023101 & 256.324369907 \\
\hline
\end{tabular}

with eight complex second-largest roots. The secular equation has a branch $x=\omega z^{-6}$ with $\omega^{8}=1, \omega=e^{2 \pi i p / 8}$. The primitives $(p=1,3,5,7)$ give four roots

$$
\begin{aligned}
& \bar{x}(p)=w z^{-6}-\frac{1}{2}\left(1-w^{6}\right) z^{-4}+\frac{1}{2} w z^{-2}-\frac{17}{32} w z^{2}+\frac{3}{2}\left(1-w^{6}\right) z^{4} \\
& +\frac{337}{128} w z^{6}+4\left(1-w^{6}\right) z^{8}+\frac{21915}{2048} w z^{10}+\cdots
\end{aligned}
$$

all of which have equal magnitude real and imaginary parts. The roots for which $p=0,4(\omega= \pm 1)$ give eigenvalues

$$
\begin{aligned}
\bar{x}(p)=\omega z^{-6}+z^{-4}+\frac{3}{2} \omega z^{-2}+ & 4+\frac{67}{8} \omega z^{2} \\
& +15 z^{4}+\frac{359}{16} \omega z^{6}-10 z^{8}-\frac{15261}{128} \omega z^{10}+\cdots
\end{aligned}
$$

and $p=2,6(\omega= \pm i)$ result in polynomials

$$
\bar{x}(p)=\omega\left(z^{-6}+z^{-2}-z^{2}-z^{6}\right)
$$

The $4 \times N$ Baxter-Wu ribbon is interesting for having its four largest-magnitude eigenvalues all of the same leading order in $z$, the first level has four branches $Q=\left(x z^{6}\right)^{4}-\left(x z^{6}\right)^{3}-\left(x z^{6}\right)^{2}+1$, or $x=z^{-6}$, $x=\omega z^{-6}$ with $\omega=e^{2 \pi i p / 3}, p=0,1,2$;

$$
\begin{aligned}
& \bar{x}_{\text {max }}=\bar{x}_{1}=z^{-6}+4+10 z^{2}+30 z^{4}+78 z^{6}+174 z^{8}+307 z^{10}+\cdots \\
& \bar{x}_{2,3,4}=\omega\left(z^{-6}+\frac{4}{3}-\frac{2}{3} z^{2}-2 z^{4}-\frac{34}{9} z^{6}-\frac{26}{9} z^{8}+\frac{59}{9} z^{10}+\cdots\right)
\end{aligned}
$$

In all of the examples in this subsection, there is agreement with purely numerical determination of the eigenvalues for $z=e^{\beta J}=0.1,0.2$ to ten decimal places or more, for both real and imaginary parts. Our purpose here is not to tout the precision (but it is a welcome feature of the results), but rather to point out that our methods do not suffer from any of the problems associated with eigenvalue degeneracy or neardegeneracy that plague many purely numerical approaches.

\section{Conclusions and Conjectures}

The Newton polygon algorithm offers some distinct advantages for the study of nanotubes and ribbons. In the first place series expansions for all eigenvalues of the transfer matrix can be found 


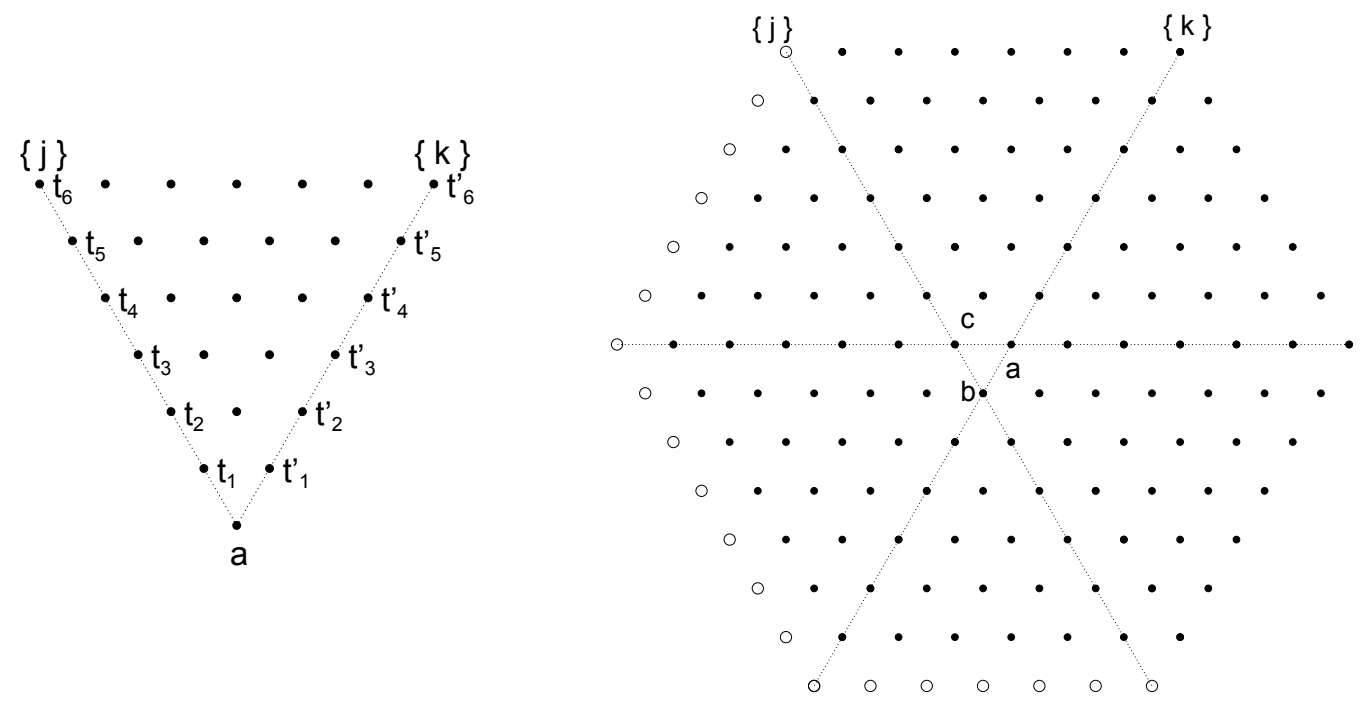

Figure 8: Graphical representation of $A(a)=A_{\mathbf{k}, \mathbf{j}}(a)$ (left) and $Z_{w}$ (right).

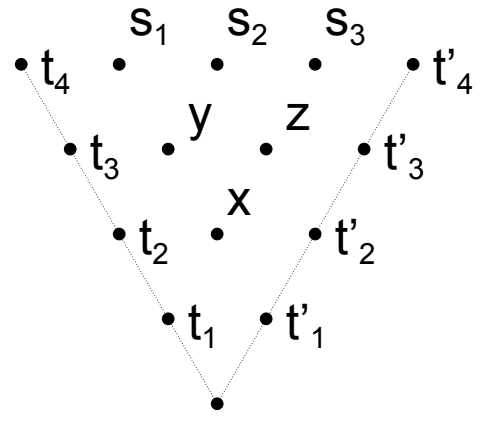

a

Figure 9: An explicit case of $A(a)$ for $N=4$.

symbolic-numerically: as functions of some quantity such as fugacity or a Boltzmann weight. Both very large and very low fugacity regimes, and the cases of ferromagnetic or anti-ferromagnetic systems, are equally approachable. Secondly the technique is not an approximation. Each iteration gives the next order in the series expansion exactly. Furthermore if a root truncates to a finite sum of terms, the Newton polygon process itself simply halts, no new segments are produced once the last term in the series is reached. A third point is that degeneracy of a root presents none of the problems normally encountered by numerical root extraction methods in this case. The level of degeneracy of a root has tell-tale signs in the calculation.

The technique is not limited to a single expansion variable $z$, Inaba [19] has used an extended Hensel construction based upon the Newton polygon algorithm to factor multivariate polynomials.

\section{Leading exponents}

There appears to be a correlation between the leading terms of eigenvalues, the number of eigenvalues with this leading term, and the trace of the transfer matrix for the ferromagnetic Ising tubes.

\begin{tabular}{|l|l|l|}
\hline \multicolumn{2}{|l|}{ Ferromagnetic Ising } & $\boldsymbol{m} \times \boldsymbol{N}$ tube \\
\hline 3 & $\begin{array}{l}\text { Level } 1 \text { leading term } \\
\text { (degeneracy) }\end{array}$ & $\operatorname{Tr}(T)$ \\
\hline 3 & $\begin{array}{l}z^{6}(2), \\
z^{2}(6)\end{array}$ & $2 Z^{2}\left(3+z^{4}\right)$ \\
\hline 4 & $\begin{array}{l}z^{8}(2), \\
z^{4}(12),\end{array}$ & $2\left(1+6 z^{4}+z^{8}\right)$ \\
\hline 5 & $z^{0}(2)$ & \\
\hline 5 & $\begin{array}{l}z^{10}(2), \\
z^{6}(20),\end{array}$ & $2 z^{2}\left(z^{8}+10 z^{4}+5\right)$ \\
\hline & $z^{2}(10)$ & \\
\hline 6 & $\begin{array}{l}z^{12}(2), \\
z^{8}(30), \\
z^{4}(30),\end{array}$ & $2\left(z^{12}+15 z^{8}+15 z^{4}+1\right)$ \\
$z^{0}(2)$ & \\
\hline
\end{tabular}

The trace is rather easy to calculate in the general case $(m \times N$ Ising tube):

$$
\begin{aligned}
& \operatorname{Tr}(T)=\sum_{s_{1}= \pm 1} \cdots \sum_{s_{m}= \pm 1} z^{\sum_{i=1}^{m} s_{i}^{2}+\sum_{i}^{P B C} s_{i} s_{i+1}} \\
& =z^{m} \sum_{s_{1}= \pm 1} \cdots \sum_{s_{m}= \pm 1} \prod_{i}^{P B C} z^{s_{i} s_{i+1}} \\
& =z^{m} \sum_{s_{1}= \pm 1} \cdots \sum_{s_{m}= \pm 1}^{P B C}\left(\cosh (\beta J)+s_{i} s_{i+1} \sinh (\beta J)\right), \quad z=e^{\beta J} \\
& =z^{m} 2^{m}\left(\cosh ^{m}(\beta J)+\sin ^{m}(\beta J)\right)=\left(z^{2}+1\right)^{m}+\left(z^{2}-1\right)^{m}
\end{aligned}
$$

For hard squares we can make no such conjecture, since the trace of the transfer matrix (the sum of Boltzmann weights for all allowed configurations with adjacent "rungs" of the ladder in identical states) is simply one for all $m$.

\begin{tabular}{|l|l|c|}
\hline \multicolumn{2}{|l|}{ Hard squares $\boldsymbol{m} \times \boldsymbol{N}$ tube } \\
\hline$m$ & Level 1 leading term (degeneracy) & $\operatorname{Tr}(T)$ \\
\hline 4 & $0(9), z^{0}(1), z^{1}(4), z^{2}(2)$ & 1 \\
\hline 5 & $0(21), z^{0}(1), z^{1}(5), z^{2}(5)$ & 1 \\
\hline 6 & $0(46), z^{0}(1), z^{1}(6), z^{2}(9), z^{3}(2)$ & 1 \\
\hline 7 & $z^{0}(1), z^{1}(7), z^{2}(14), z^{3}(7)$ & 1 \\
\hline 8 & $0(209), z^{0}(1), z^{1}(8), z^{2}(20), z^{3}(16), z^{4}(2)$ & 1 \\
\hline
\end{tabular}




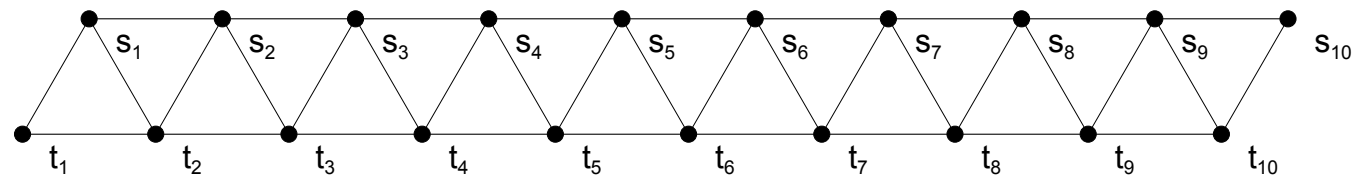

Figure 10: Two rows of the lattice, with sites labeled appropriately for constructing the row-to-row transfer matrix.

\section{References}

1. RJ Baxter (1982) Exactly Solved Models in Statistical Mechanics. Dover, New York.

2. Domb C, Green MS (1974) Phase Transitions and Critical Phenomena (Volume 3). Series Expansions for Lattice Models. Academic Press, London.

3. Jaan Oitmaa, Chris Hamer, Weihong Zheng (2006) Series Expansion Methods For Strongly Interacting Lattice Models. Cambridge University Press, Cambridge.

4. Baxter RJ (1981) Corner transfer matrices. Physica 106A: 18-27.

5. Baxter RJ (2007) Corner transfer matrices in statistical mechanics. J Phys A Math Theor40: 12577-12588.

6. Celine Cattoen, Matt Visser (2006) Generalized Puisieux series expansion for cosmological milestones. arXiv:gr-qc/0609073.

7. Takua Kitamoto (1994) Approximate eigenvalues, eigenvectors and inverse of a matrix with polynomial entries. J Indust Appl Math 11: 73-85.

8. Takeaki Sasaki, Fujio Kako (1999) Solving multivariate algebraic equation by Hensel construction.J Indust Appl Math16: 257-285.

9. David A Cox, John Little, Donal O'Shea (2005) Using Algebraic Geometry (2nd edn). Springer.
10. Robert JW (1950) Algebraic Curves. Dover, New York.

11. S Basu, R Pollack, Marie-Françoise, C Roy (2006) Algorithms in Real Algebraic Geometry (2nd edn), Springer.

12. Kung HT, Traub JF (1978)All Algebraic Functions Can Be Computed Fast. JACM 25: 245-260.

13. Thomas H Cormen, Charles E Leisterson, Ronald L Rivest, Clifford Stein (2009) Introduction to Algorithms ( $3^{\text {rd }}$ edn). The MIT Press, Cambridge.

14. http://reduce-algebra.sourceforge.net/.

15. http://www.singular.uni-kl.de

16. Baxter RJ (1999) Planar lattice gases with nearest-neighbor exclusion. Annals of Combinatorics 3: 191-203.

17. David Cox, John Littel, Donal O'Shea (1997) Ideals, Varieties and Algorithms. Springer, New York, USA.

18. Takua Kitamoto (1994) Approximate eigenvalues, eigenvectors and inverse of a matrix with polynomial entries. J Indust Appl Math 11: 73-85.

19. Daiju Inaba (2005) Factorization of multivariate polynomials by extended Hensel construction. ACM SIGSAM Bulletin. 39: 2-14. 